\title{
B lymphocytes confer immune tolerance via cell surface GARP-TGF- $\beta$ complex
}

\author{
Caroline H. Wallace, ${ }^{1}$ Bill X. Wu, ${ }^{1}$ Mohammad Salem, ${ }^{1}$ Ephraim A. Ansa-Addo, ${ }^{1}$ Alessandra Metelli, ${ }^{1}$ \\ Shaoli Sun, ${ }^{2}$ Gary Gilkeson, ${ }^{1,3}$ Mark J. Shlomchik, ${ }^{4}$ Bei Liu, ${ }^{1}$ and Zihai Li ${ }^{1,5}$ \\ ${ }^{1}$ Department of Microbiology and Immunology, ${ }^{2}$ Department of Pathology and Laboratory Medicine, and ${ }^{3}$ Department of \\ Medicine, Medical University of South Carolina, Charleston, South Carolina, USA. ${ }^{4}$ Department of Immunology, University \\ of Pittsburgh, Pittsburgh, Pennsylvania, USA. ${ }^{5}$ First Affiliated Hospital, Zhengzhou University School of Medicine, \\ Zhengzhou, China.
}

\begin{abstract}
GARP, a cell surface docking receptor for binding and activating latent TGF- $\beta$, is highly expressed by platelets and activated Tregs. While GARP is implicated in immune invasion in cancer, the roles of the GARP-TGF- $\beta$ axis in systemic autoimmune diseases are unknown. Although $B$ cells do not express GARP at baseline, we found that the GARP-TGF- $\beta$ complex is induced on activated human and mouse $B$ cells by ligands for multiple TLRs, including TLR4, TLR7, and TLR9. GARP overexpression on B cells inhibited their proliferation, induced IgA class-switching, and dampened $T$ cell-independent antibody production. In contrast, B cell-specific deletion of GARP-encoding gene Lrrc32 in mice led to development of systemic autoimmune diseases spontaneously as well as worsening of pristane-induced lupus-like disease. Canonical TGF- $\beta$ signaling more readily upregulates GARP in Peyer patch $B$ cells than in splenic $B$ cells. Furthermore, we demonstrated that $B$ cells are required for the induction of oral tolerance of $T$ cell-dependent antigens via GARP. Our studies reveal for the first time to our knowledge that cell surface GARP-TGF- $\beta$ is an important checkpoint for regulating $B$ cell peripheral tolerance, highlighting a mechanism of autoimmune disease pathogenesis.
\end{abstract}

Conflict of interest: The authors have declared that no conflict of interest exists.

Submitted: January 16, 2018

Accepted: March 6, 2018

Published: April 5, 2018

Reference information: JCI Insight. 2018;3(7):e99863. https://doi.org/10.1172/jici. insight.99863.

\section{Introduction}

Glycoprotein A repetitions predominant (GARP), encoded by the gene Lrrc32, is a type 1 transmembrane protein that serves as the cell surface docking receptor for latent transforming growth factor- $\beta$ (LTGF- $\beta$ ) (1, 2). LTGF- $\beta$ exists in abundance on the surface of Tregs and platelets via binding to the extracellular region of GARP (2-7). Multiple studies in Tregs have demonstrated that GARP promotes TGF- $\beta$ biogenesis and LTGF- $\beta$ activation (8-10). Further, GARP expression has been observed in cells with heightened TGF- $\beta$ activity: hepatic stellate cells (11), mesenchymal stromal cells (12), and multiple aggressive cancer types (13-15).

TGF- $\beta$ is a master cytokine for maintaining immunological tolerance: early studies demonstrated that TGF- $\beta 1^{-/-}$mice develop lethal multiorgan inflammatory disease with a high level of autoantibodies, including antinuclear antibody (ANA) $(16,17)$. TGF- $\beta$ biology has been extensively characterized, and the molecule exists in various biochemical forms: active and free soluble TGF- $\beta$; LTGF- $\beta$ formed by TGF- $\beta$ associated with latency-associated peptide (LAP); LTGF- $\beta$ in complex covalently with large TGF- $\beta$-binding protein (LTBP); and the membrane form of LTGF- $\beta$ (mLTGF- $\beta$ ) in association with GARP (18-20). Only LAP-free soluble TGF- $\beta$, activated by both integrin-dependent and -independent mechanisms, is biologically active $(21,22)$.

Although TGF- $\beta$ functions through both canonical and noncanonical signaling pathways, B cells require the canonical pathway for TGF- $\beta$-induced apoptosis $(23,24)$. TGF- $\beta$ signaling commences at the cell surface via type I and type II TGF- $\beta$ receptors, leading to downstream Smad2 phosphorylation (25). While B cells utilize both paracrine and autocrine TGF- $\beta$ signaling, prior studies suggest that TGF- $\beta$ regulation of apoptosis in B cells is an autocrine mechanism $(24,26)$. However, it is not clear what cell-intrinsic mechanisms B cells use to self-regulate TGF- $\beta$-induced apoptosis. Additional roles of TGF- $\beta$ signaling in $\mathrm{B}$ cells include (a) induction of class-switch recombination (CSR) of Ig to IgA during the differentiation of mature B cells to IgA-producing plasma cells and (b) B cell regulation through cell autonomous production of TGF- $\beta 1$ (27-29). Using a Cre-loxP system, Cazac and Roes demonstrated that Tgfbr2 $2^{-1}$ conventional B 
cells had a reduced life span, while there was expansion of $T g f b r 2^{-1-}$ peritoneal B-1 cells, B cell hyperplasia in Peyer's patches (PPs), and elevated serum Ig. Notably, these mice had severe IgA deficiency (30). This body of work highlights the critical functions of TGF- $\beta$ in B cell biology.

Both central and peripheral tolerance checkpoints are necessary to prevent B cell-driven autoimmunity (31-33). Recent work on GARP in the context of TGF- $\beta$ has attempted to address the role of GARP in promoting a tolerogenic environment $(6,34-36)$. However, no study has addressed the physiologic role of GARP in tolerance in vivo. In particular, the roles of GARP in the context of B cell-driven autoimmune diseases are unknown. In this study, we found that B cells express GARP on the cell surface in response to multiple TLR ligands. We studied the mechanism of GARP induction as well as the immunological relevance of B cell-intrinsic GARP in immune tolerance using both gain- and loss-of-function studies. We uncovered, for the first time to our knowledge, that the B cell GARP-LTGF- $\beta$ axis serves as a vital immune checkpoint for B cell tolerance and prevention of lupus-like autoimmune diseases in mice.

\section{Results}

TLR activation of both murine and human B cells induces cell surface GARP and LTGF- $\beta$ expression. TLRs are key innate immune receptors that sense pathogen-associated molecular patterns and regulate activation of immune responses. Activation of TLRs on B cells directly induces B cell proliferation and enhances antigen presentation, cytokine secretion, plasma cell differentiation, CSR, and memory B cell differentiation (37, 38). Past research has extensively demonstrated a significant connection between heightened TLR activity and autoimmune diseases (39-43).

We hypothesized that TLR stimulation turns on negative regulatory pathways, such as GARP-LTGF- $\beta$, to prevent B cell hyperactivation. To this end, CD19+ B cells were isolated from WT mouse spleens and cultured for $24-120$ hours with anti- $\mu$ antibody $(15 \mu \mathrm{g} / \mathrm{ml})$ for B cell receptor $(\mathrm{BCR})$ stimulation or various TLR ligands: LPS (TLR4 ligand), R848 (TLR7/8 ligand), and CpG (TLR9 ligand). We found that multiple TLR ligands potently induced cell surface expression of GARP and LTGF- $\beta$, while BCR stimulation only induced modest GARP expression (Figure 1A). The kinetics of GARP surface expression differed depending upon the TLR ligands used (Figure 1B). GARP upregulation was also confirmed by immunoblot (Figure 1C). Interestingly, we found that ligation of the MyD88-independent TLR3 did not induce surface GARP expression, but a combination of IL-1 $\beta$ and Poly I:C did, compared with GARP-KO $B$ cells as negative control (Figure 1D). Phenotypic analysis of LPS-treated splenic CD $19^{+}$B cells revealed that $\mathrm{GARP}^{+}$cells were larger (increase in FSC and SSC) and more highly activated than GARP ${ }^{-}$cells, with significantly higher levels of CD86, CD80, and CD44. Levels of CD23 and PD-L1 were also significantly elevated, while no change was seen in response to LPS for the expression of IgM, CD62L, MHC class II, CD1d, and PD-1 (Figure 1E).

Similar to murine B cells, human $\mathrm{CD} 19^{+} \mathrm{B}$ cells upregulated GARP in response to both R848 (TLR7/8 ligand) and CpG (TLR9 ligand). However, distinct from mouse B cells, BCR stimulation also upregulated surface GARP and LAP on human B cells as was described recently (44), although at lower levels than TLR signaling (Figure 1, F and G). Both murine and human B cells upregulated GARP in response to TLR stimuli, but it is not known how TLR-induced GARP expression regulates B cell functions. As GARP is necessary for the surface expression and activation of LTGF- $\beta(4,5)$, our findings suggest that B cell GARP expression in response to TLR activation may be an important negative checkpoint for B cell activation (41).

GARP overexpression on B lymphocytes reduces proliferation, increases IgACSR, and attenuates T cell-independent antibody production. In order to understand the biological significance of B cell GARP expression, we generated an inducible mouse model to control GARP expression pharmacologically (45). We knocked in a Tet-on trans-acting element to the promoter region of the endogenous GARP gene, Lrrc32. Crossing of Lrrc32 ${ }^{\text {TetO }}$ mice with Rosa26 $6^{t T A}$ mice allowed inducible GARP overexpression (OE) using doxycycline (Figure 2A). If the primary function of GARP in B cells is to regulate TGF- $\beta$ activation and availability, then transgenic OE of GARP is hypothesized to alter IgA CSR, B cell proliferation, and antibody responsiveness $(27,29)$.

CD19 microbead-purified splenic B cells from WT or GARP OE mice were stimulated with anti- $\mu$ antibody, LPS, or a combination of anti- $\mu$ antibody, CD40L, and LPS, in the presence of doxycycline. Higher GARP expression was sustained on GARP OE B220+ splenic B cells for at least 96 hours (Figure $2 \mathrm{~B}$ ). Consistent with the roles of GARP in activating TGF- $\beta$, GARP OE cells proliferated less than WT cells after stimulations, based on CFSE dilution and cell count (Figure 2, C and D). The IgA level was significantly increased in GARP OE B cell cultures in all conditions, including treatment with 


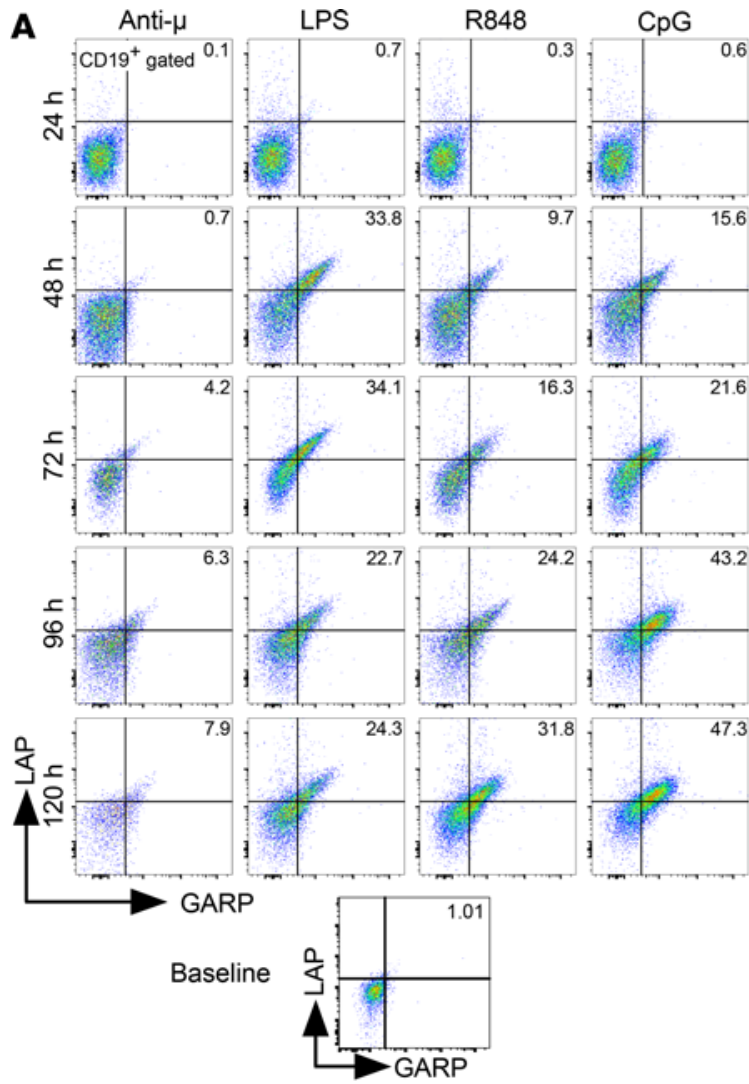

D
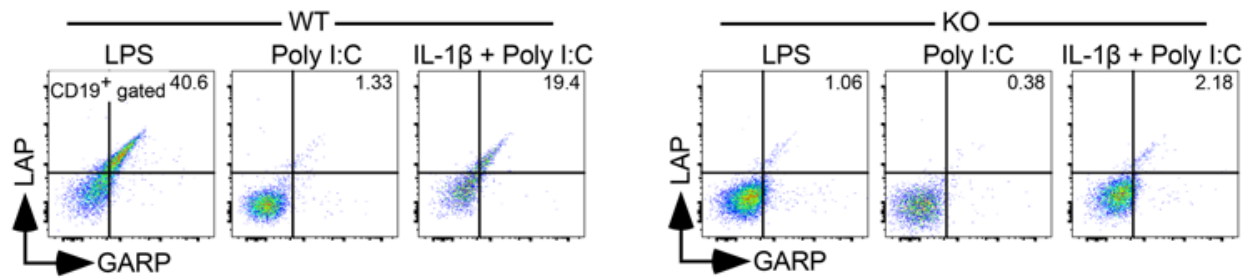

E
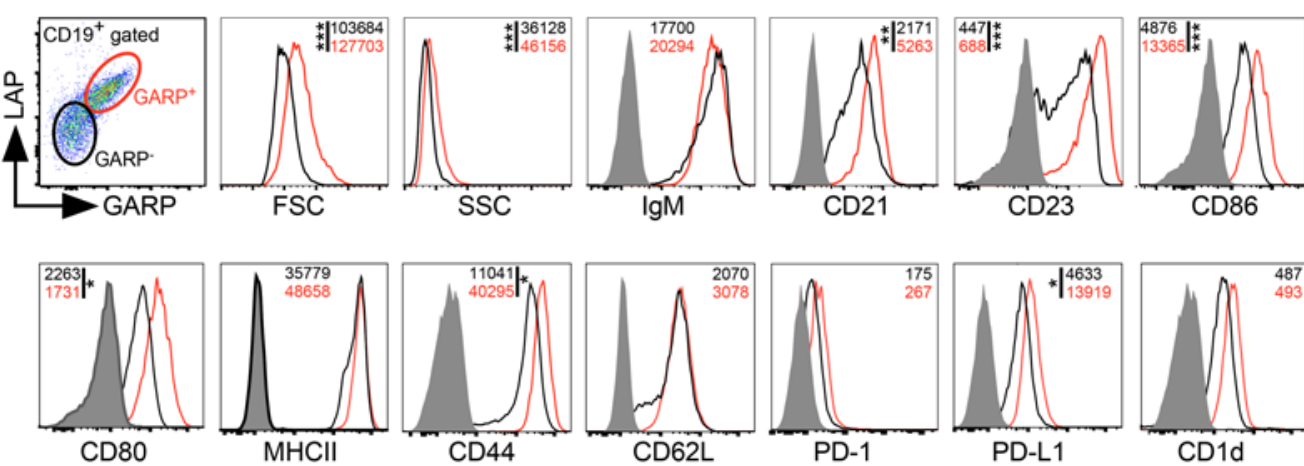

F
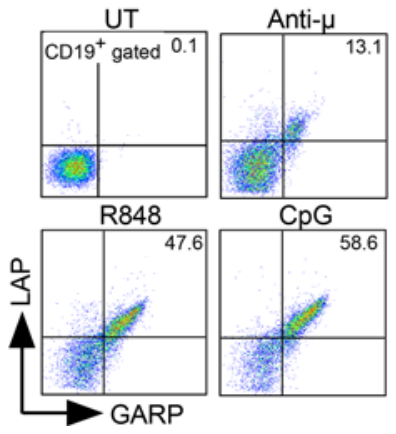

G

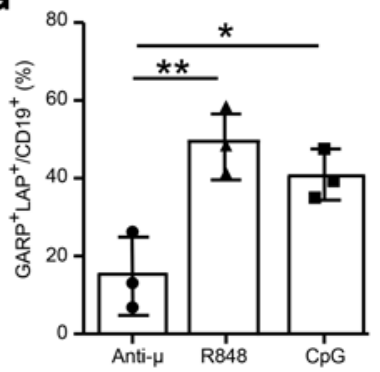

Figure 1. Multiple TLR ligands induce GARP expression on both human and mouse $B$ cells. Splenic B cells were isolated from WT mouse spleens using CD19+ magnetic beads. (A) Cells were stimulated with mouse anti- $\mu$ $(15 \mu \mathrm{g} / \mathrm{ml})$, LPS, R848, or CpG for 120 hours. Flow cytometry was used at 24-hour intervals to measure GARP+ $\mathrm{LAP}^{+}$expression on live $B$ cells. Numbers represent the percentage of cells in the GARP'LAP+ quadrant over the entire CD19+ B cell population. Flow plots are representative of 3 independent experiments. A representative baseline flow plot is also shown. (B) Quantification of GARP and LAP expression ( $n$ $=4$ biological replicates). MFI, mean fluorescent intensity of GARP. Statistical analysis was performed by 2-way ANOVA ${ }^{* *} P<0.001$. (C) Immunoblot of GARP in the whole-cell lysate of untreated (UT) WT B cells or after stimulation with the indicated conditions for 72 hours. Representative of 3 immunoblots. (D) Primary WT and GARP-KO splenic $B$ cells were cultured with LPS, Poly I:C, or IL-1 $\beta$ plus Poly I:C for 72 hours. Cells were stained for GARP and LAP and analyzed by flow cytometry. Representative of 3 independent experiments. (E) Phenotypic analysis of LPS-treated (48 hours) GARP- $^{-}$and GARP' $B$ cells by flow cytometry. Histogram plots are representative of $n=3$ biological repeats and 2 independent experiments. Black lines denote GARP cells, red lines denote GARP ${ }^{+}$ cells; shaded areas denote isotype. Numbers represent mean fluorescent intensity (MFI). (F) Human B cells were isolated from normal subjects using human anti-CD19 ${ }^{+}$magnetic beads. Cells were freshly analyzed or cultured with human anti- $\mu, \mathrm{R} 848$, or $\mathrm{CpG}$ for 72 hours. GARP+LAP+ levels were analyzed by flow cytometry. Representative of 3 independent experiments. (C) Quantification of GARP+LAP' expression in 3 biological replicates from healthy donors. Each data point represents an individual donor. Statistical analysis was performed by 2-tailed $t$ test (E) and 1-way ANOVA with Tukey's multiple comparisons $(\mathbf{G}) ;{ }^{*} P<0.05,{ }^{*} P$ $<0.01,{ }^{* *} P<0.001$. Error bars represent SD. 
A

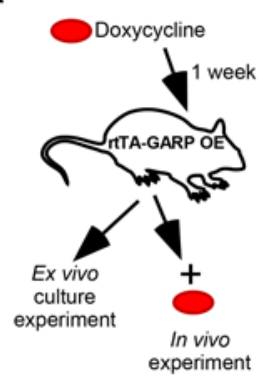

B
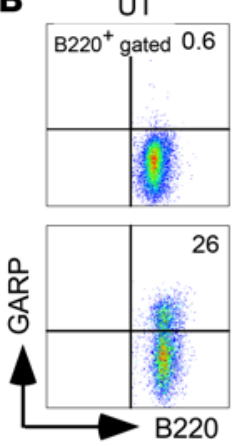

C

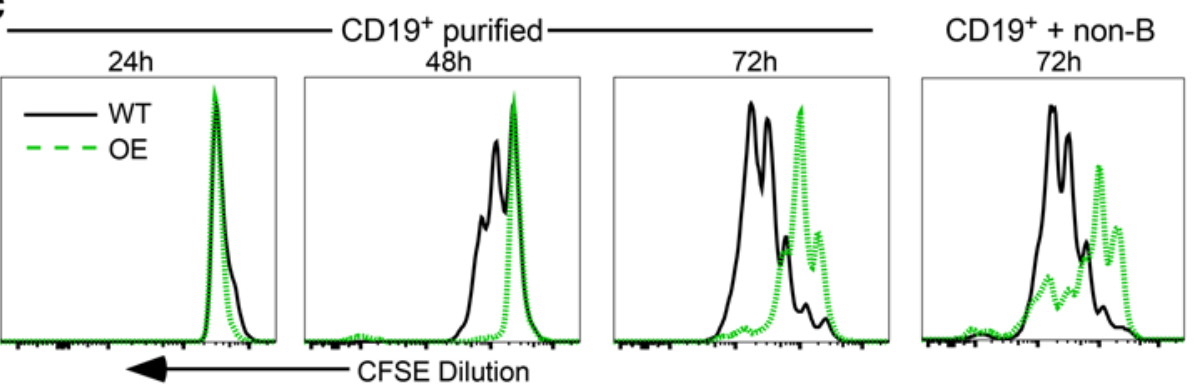

D

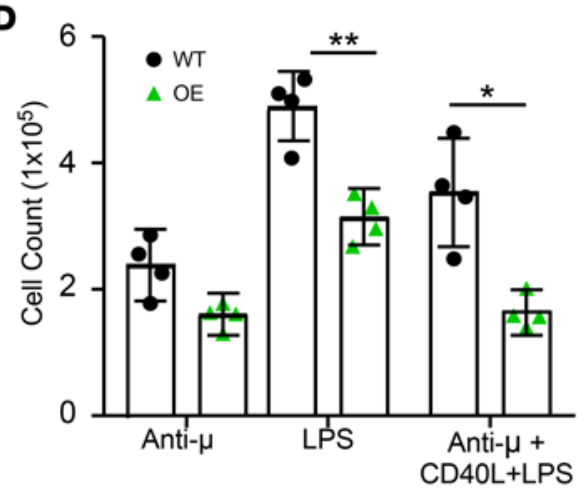

$\mathbf{F}$

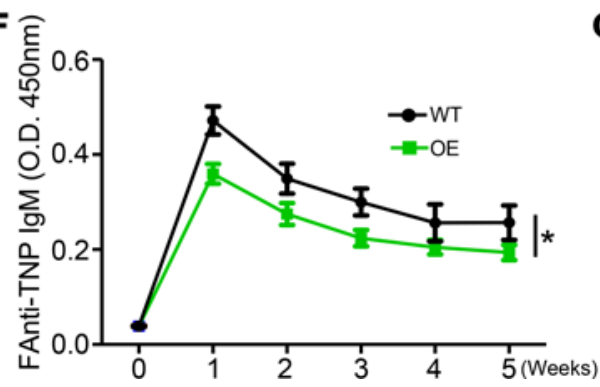

E

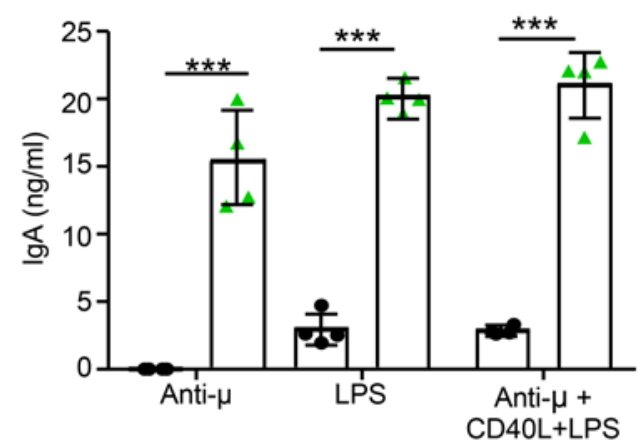

G

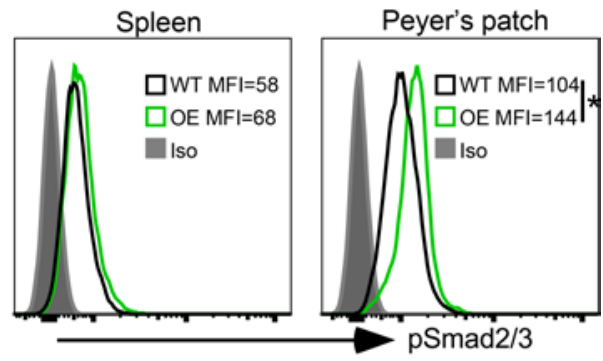

Figure 2. GARP overexpression dampens B cell proliferation and alters antibody production. rtTA GARP OE mice were given doxycycline to induce GARP expression broadly. (A) Diagram of the experiment scheme.

(B) Analysis of GARP and LAP on WT and GARP OE splenic CD19 bead-purified B cells immediately ex vivo (UT) and after 96-hour treatment with anti- $\mu$ antibody, LPS, or a combination of anti- $\mu$ antibody, CD4OL, and LPS. Numbers represent percentage of ${\mathrm{B} 22 \mathrm{O}^{+} \mathrm{CARP}}^{+}$cells over the gated CD19+ $B$ cell population. Flow plots are representative of $n=4$ biological replicates. (C) WT and $\mathrm{OE}$ splenic $\mathrm{CD19}{ }^{+}$purified $\mathrm{B}$ cells were labeled with CFSE and cultured for 3 days with LPS. CFSE dilution was measured by flow cytometry at 24-hour intervals. CD19+ purified CFSE-labeled $B$ cells were also cultured with WT non-B cell spleen cells for 72 hours, and CFSE dilution was assessed by flow cytometry. Histograms are representative of 2 independent experiments. (D) Live cell count of 96-hour stimulated cells was analyzed by trypan blue exclusion $(n=4)$. (E) Total IgA levels in the 96-hour supernatants were measured by ELISA $(n=4)$. (F) WT and CARP OE mice were given doxycycline in the drinking water for 1 week prior to immunization with TNP-ficoll, and this was continued throughout the experiment. TNP-specific IgM levels were measured in the sera of indicated mice ( $n=6 \mathrm{WT}$ and $n=7$ OE biological replicates). Statistics were performed by 2-way ANOVA; ${ }^{*} P<0.05$. (C) Splenic and Peyer's patch $\mathrm{CD}_{19} 9^{+}$bead-purified $\mathrm{B}$ cells were isolated from doxycycline-treated WT and CARP OE mice and immediately stained and assessed for intracellular $\mathrm{pSmad} 2 / 3$ levels using flow cytometry ( $n=3$ biological replicates). Representative of 3 independent experiments. Histograms depict $\mathrm{pSmad} 2 / 3$ staining; gray shaded areas represent the isotype control, black lines represent WT, and green lines represent $\mathrm{OE}$. All statistical analysis was performed by 2 -tailed $t$ test unless otherwise indicated; ${ }^{*} P<0.05,{ }^{* *} P<$ $0.01,{ }^{* *} P<0.001$. Error bars represent SD.

anti- $\mu$ antibody alone (Figure 2E). To further probe the functional significance of B cell GARP in vivo, we immunized WT and GARP OE mice with a T cell-independent antigen, 2,4,6-Trinitrophenyl-AECM-ficoll (TNP-ficoll). Consistent with the known TGF- $\beta$-induced dampening of B cell responsiveness (46), we found that GARP OE mice had a reduction in TNP-specific IgM level after immunization (Figure 2F). Further, we assessed the phosphorylation status of Smad2 and Smad3 in the spleen and PP $B$ cells ex vivo and found that GARP OE B cells had a significant increase in pSmad2/3 levels (Figure $2 \mathrm{G})$. Taken together, we conclude that GARP expression on B cells faithfully recapitulates the biology of heightened TGF- $\beta$ signaling.

$B$ cell GARP protects peripheral tolerance and prevents autoimmunity. Our findings thus far raised an intriguing possibility that the B cell GARP-LTGF- $\beta$ axis serves as a checkpoint to prevent TLR-medi- 
A

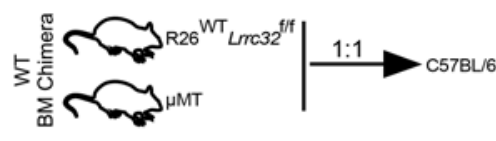

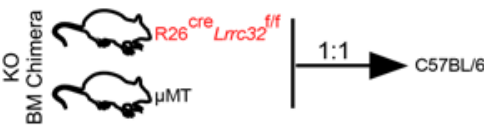

D
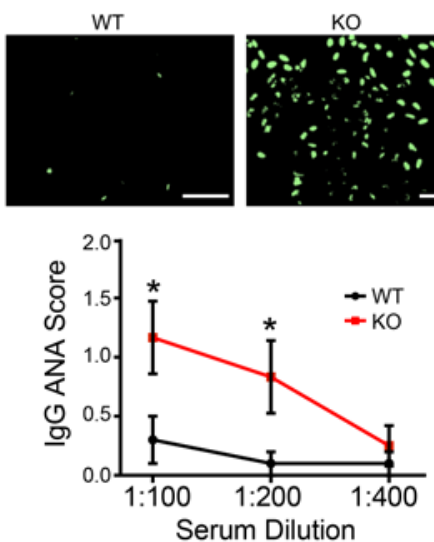

B

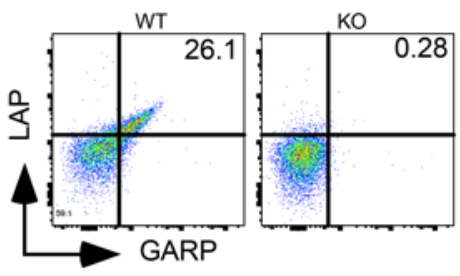

C

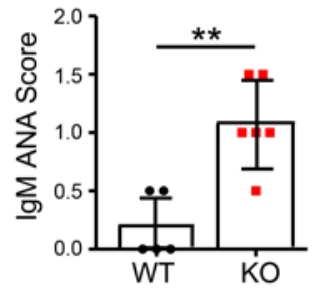

E
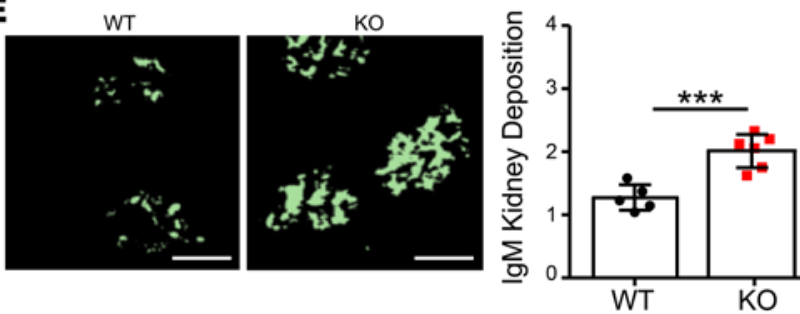

F

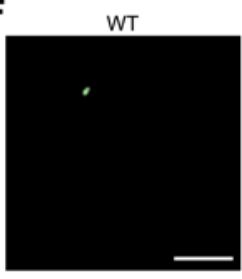

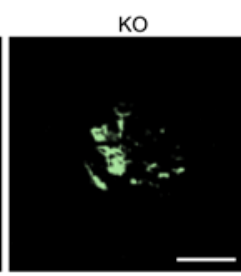

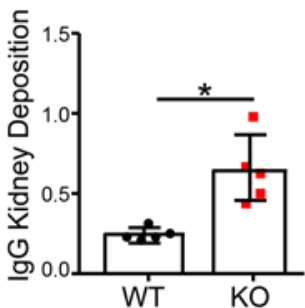

G

H
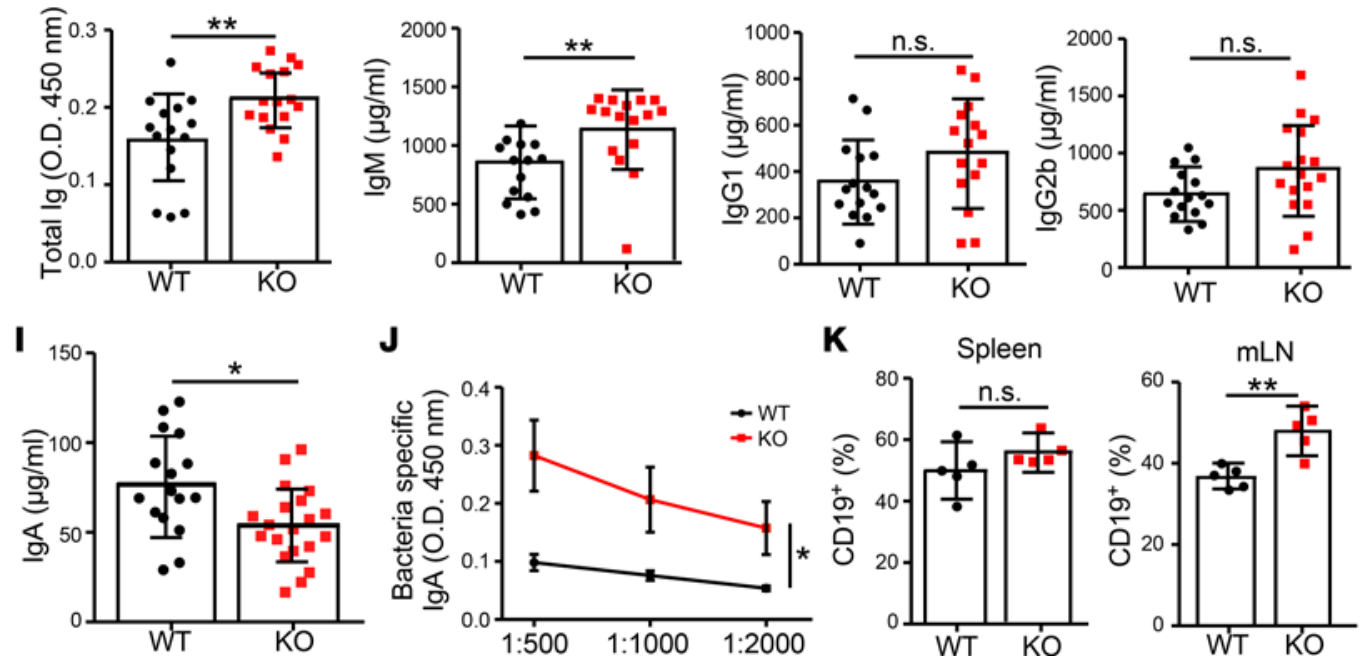

Figure 3. Mice with GARP-deficient B cells develop spontaneous lupus-like disease. (A) Schematic of the generation of B cell-specific Lrrc32-KO mice using a mixed bone marrow chimera (BM chimera) strategy. (B) Confirmation of effective GARP KO in B cells 3 months after bone marrow reconstitution. Mice were bled and PBMCs were cultured with LPS for 72 hours to induce GARP expression. GARP and LAP expression was analyzed by flow cytometry. Representative of $n=5$ biological replicates. (C) IgM-specific ANAs (1:80 dilution) were analyzed 6 months after bone marrow reconstitution ( $n=5$ WT and $n=6 \mathrm{KO}$ ). (D) IgG-specific ANAs in the sera were quantified at the indicated dilutions 6 months after bone marrow reconstitution using Hep-2 slides. Representative images are shown ( $n=5$ WT and $n=6$ KO). Scale bar: $50 \mu \mathrm{m}$. (E and F) Five- $\mu \mathrm{m}$ kidney sections were stained with anti-IgM-FITC (E) and anti-IgG-FITC (F) to detect Ig deposition in the glomeruli. Quantification and representative images are shown ( $n=5 \mathrm{WT}$ and $n=6 \mathrm{KO}$ ). Scale bar: $50 \mu \mathrm{m}$. (G) Total Ig in the sera from mice 3 months after bone marrow reconstitution was measured by ELISA ( $n=15 \mathrm{WT}$ and $n=16 \mathrm{KO})$. (H) IgM, IgG1, and IgG2b levels in the sera from mice 3 months after bone marrow reconstitution were measured by ELISA. (I) Total IgA from the sera of BM chimera mice was measured by ELISA. (J) Gut bacteria-specific IgA was measured in the sera of mice 6 months after bone marrow reconstitution $(n=5 \mathrm{WT}$ and $n=6 \mathrm{KO})$. Statistical analysis was performed by 2 -way ANOVA; ${ }^{*} P<0.05$. (K) CD19+ cells were detected in the spleen and mesenteric lymph node $(\mathrm{mLN})$ by flow cytometry $(n=5)$. All statistics performed by 2-tailed $t$ test, unless otherwise indicated; ${ }^{*} P<0.05,{ }^{* *} P<0.01,{ }^{* *} P<0.001$. Error bars represent SD. 

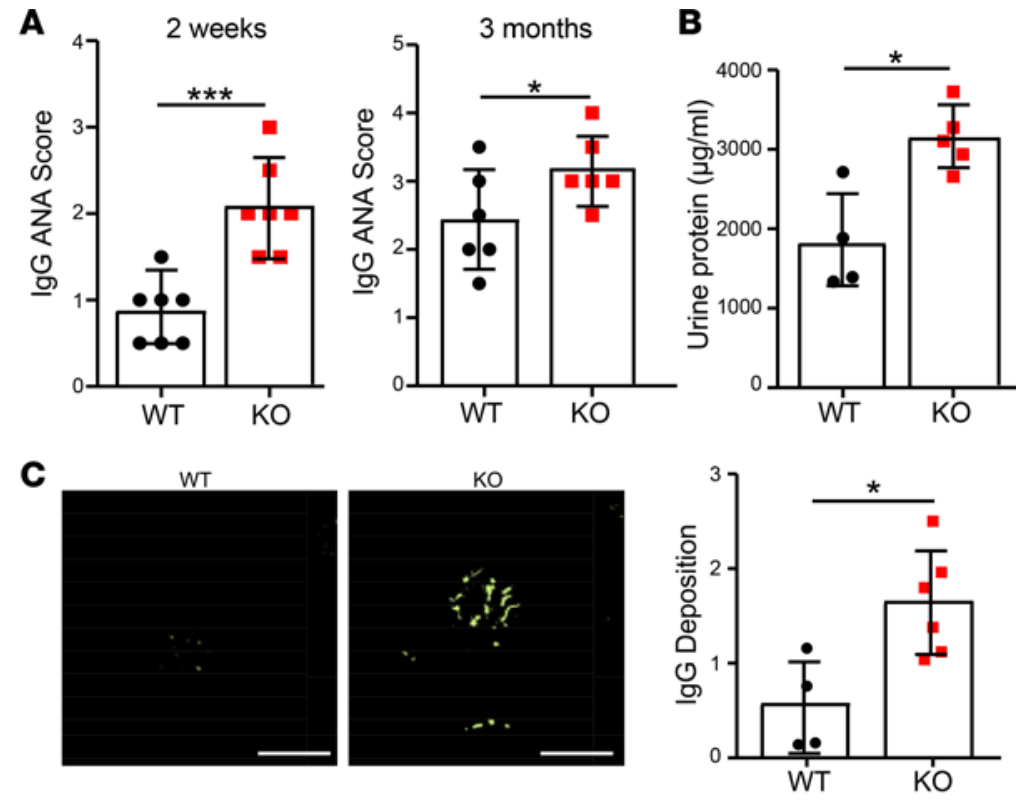

D
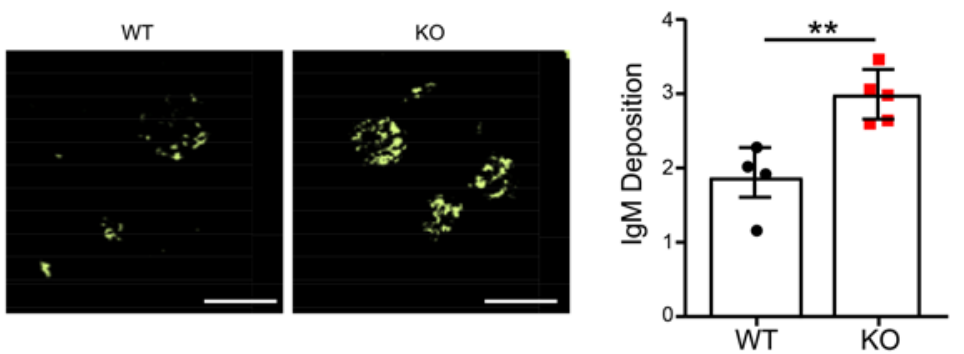

E
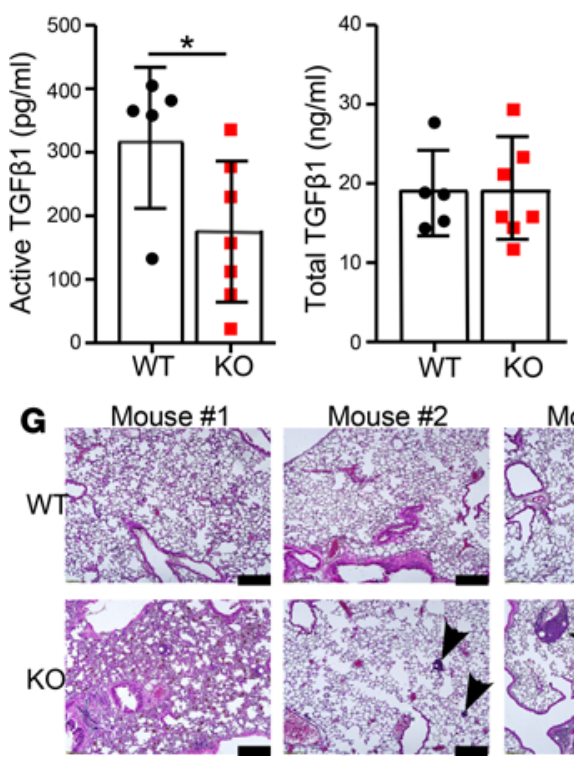

Mouse \#2
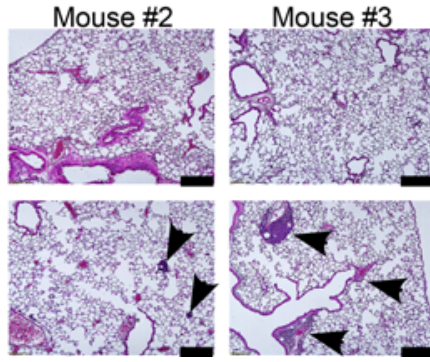

Figure 4. B cell-specific Lrrc32-KO mice have increased susceptibility to chemically induced experimental lupus. WT and B cell-specific GARP-KO bone marrow chimeric mice were injected with $500 \mu$ l pristane i.p. at 3 months after bone marrow reconstitution. Three months after pristane injection, mice were sacrificed and analyzed for the presence of lupus-like signature ( $n=6 \mathrm{WT}$ and $n=6 \mathrm{KO}$ ). (A) IgC ANAs in the serum were detected 2 weeks and 3 months after pristane injection. Serum was diluted 1:80 for 2-week analysis and 1:300 for 3-month analysis. (B) Urine protein concentration was measured by Bradford analysis from endpoint ( 3 months after pristane), with urine diluted 1:50 ( $n=4$ WT and $n=5 \mathrm{KO}$ ), as urine was not obtained from all mice. (C and D) IgC (C) and IgM (D) deposition in the kidneys was quantified. Representative images are shown ( $n=4$ WT and $n=5-6$ KO). Scale bar: $50 \mu \mathrm{m}$. (E) Active and total TGF- $\beta 1$ levels were measured in the sera of the mice using ELISA. (F) GARP and LAP expression on the surface of peripheral $B$ cells in WT and GARP-KO mice was quantified at baseline and 3 months after pristane treatment and analyzed by flow cytometry. (C) The lungs of WT and GARP-KO BM chimera mice were perfused with $4 \%$ paraformaldehyde and resected 3 months after pristane injection. Lungs were sectioned and stained with $\mathrm{H} \& \mathrm{E}$. Black arrows point to immune cell infiltrate clusters, which are quantified by pathology score. Each image is representative of 1 mouse. Scale bar: $200 \mu \mathrm{m}$. Statistical analysis was performed by 2 -tailed $t$ test; ${ }^{*} P<0.05$, ${ }^{*} P<$ $0.01,{ }^{* *} P<0.001$. Error bars represent SD.

ated autoimmune diseases. We addressed this hypothesis genetically using loss-of-function studies in B cell-specific GARP-KO mouse models. In mice, both Cd19 and Lrrc32 are located on chromosome 7, region F (47), making the use of CD19-Cre as a driver of Lrrc32 deletion difficult. To overcome this, we generated a mixed bone marrow chimera (BM chimera) model (Figure 3A). We first developed Rosa$26^{\text {cre }} \mathrm{Lrrc} 32^{f / f}$ mice and induced GARP deletion by treating the mice with tamoxifen. Rosa26 $6^{W T} \operatorname{Lrc} 32^{f / f}$ mice were used as control. Lethally irradiated WT CD45.1 C57BL6/J mice were reconstituted in a 1:1 

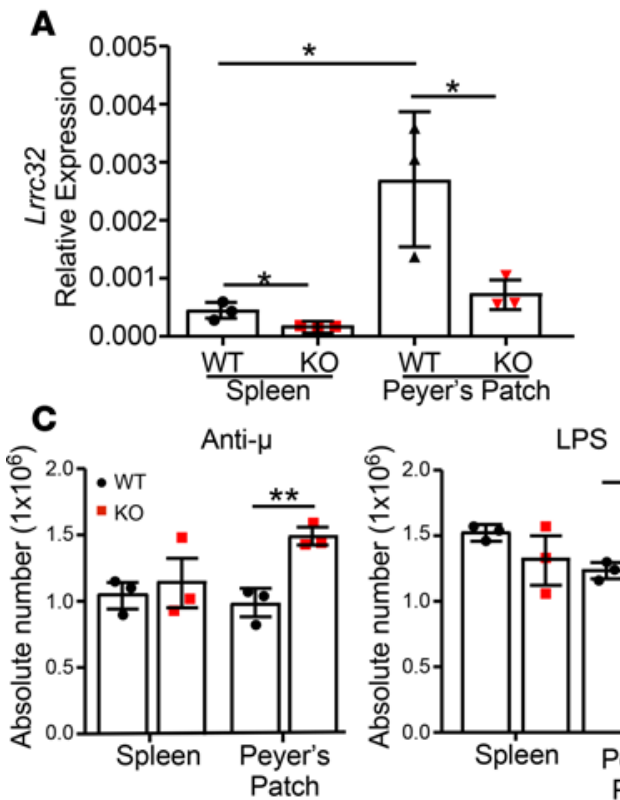

B

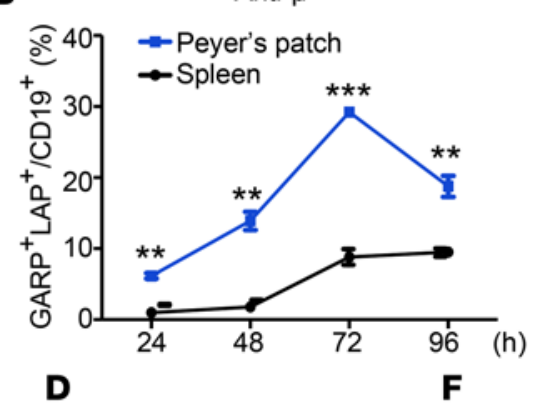

LPS

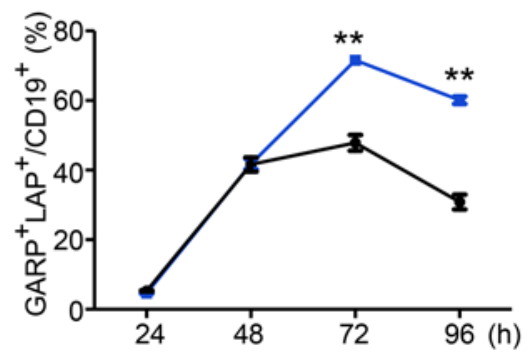

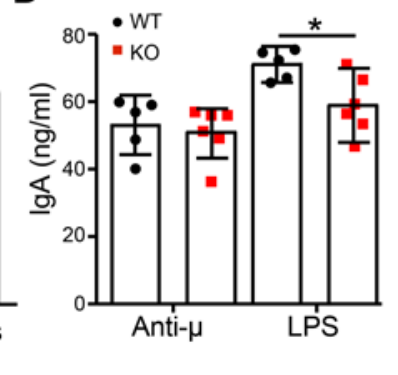
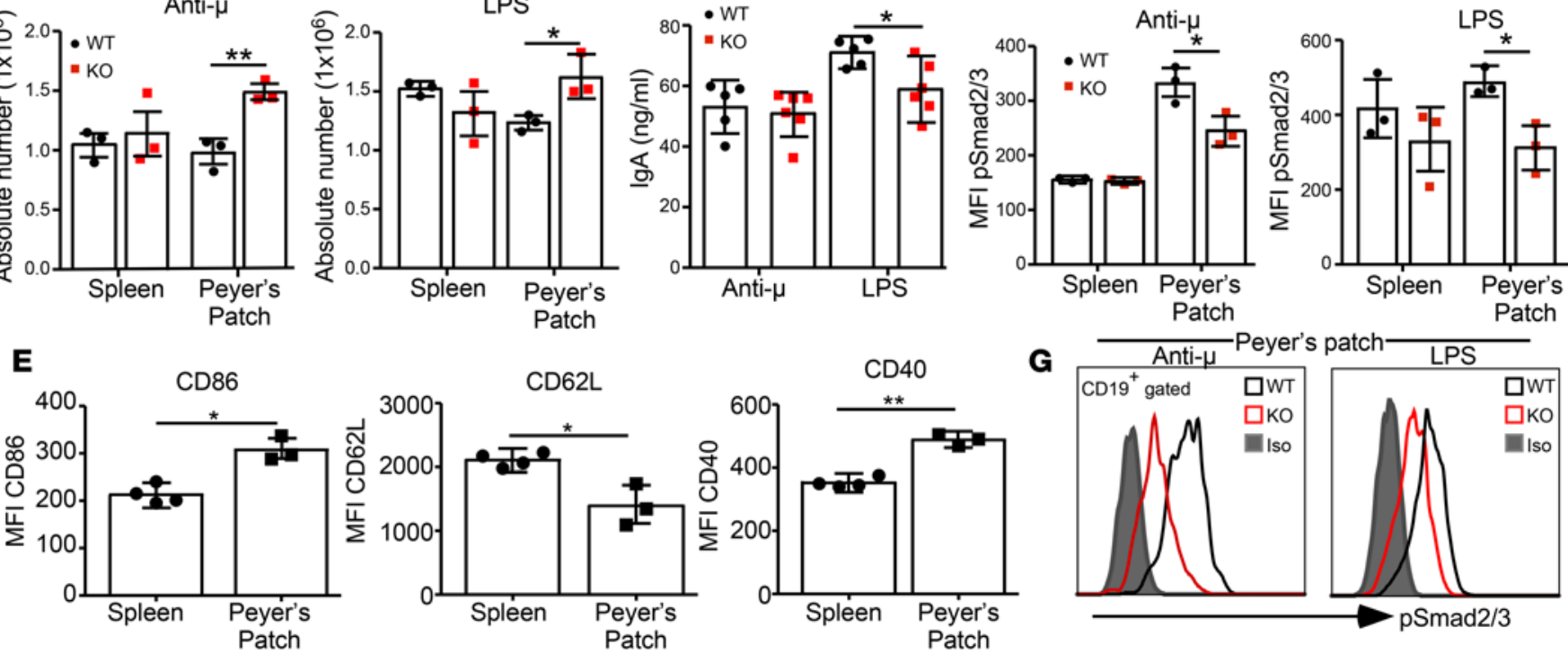

G

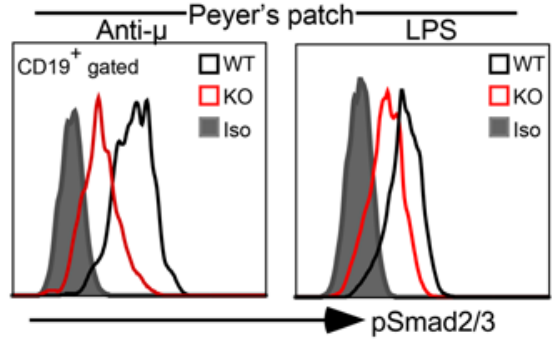

MHC Class I
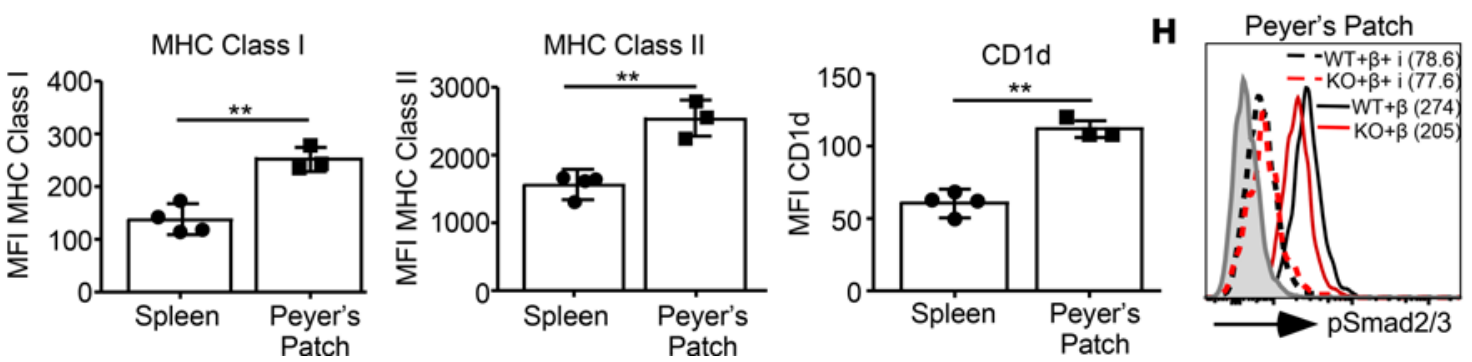

Figure 5. Peyer's patch B cells have a heightened responsiveness to the GARP-TGF- $\beta$ axis. (A) qRT-PCR analysis of $\operatorname{Lrrc} 32 \mathrm{mRNA}$ in $n=3$ biological replicates of CD19+ $B$ cells from age-matched 4-month-old WT and GARP-KO spleens and Peyer's patches. Relative expression of Lrrc32 transcripts normalized to $\beta$-actin. Representative of 2 independent experiments. (B) WT Peyer's patch and splenic CD19+ cells were cultured for 24-96 hours in the presence of anti- $\mu$ or LPS. The percentage of GARP+LAP+ cells over total CD19+ cells at each time point is quantified ( $n=3$ biological replicates and representative of 3 independent experiments). (C) Cell numbers were measured at 48 hours using trypan blue exclusion ( $n=3$ biological replicates and representative of 2 independent experiments). (D) IgA level from 96-hour cell culture of Peyer's patch B cell supernatants was quantified by ELISA ( $n=5$ WT and $n=6$ KO biological replicates combined from 2 independent experiments). (E) WT CD19+ cells were isolated from splenic and Peyer's patch compartments and stained for the indicated surface markers ( $n=$ 4 spleen and $n=3 \mathrm{PP}$ ). Each point on the graph represents an individual biological replicate. MFI, mean fluorescent intensity. (F) CD19+ B cells from spleen and Peyer's patches were treated for 96 hours with anti- $\mu$ antibody or LPS. Smad2/3 phosphorylation was measured by flow cytometry. Graphical data represent $\mathrm{pSmad2} / 3 \mathrm{MFI}$ of $n=3$ biological replicates. (C) Representative histogram of pSmad2/3 differences between WT and KO Peyer's patch B cells ( $n=3$ biological replicates representative of 2 independent experiments). (H) CD19+ $B$ cells from Peyer's patches were serum starved and treated for 1 hour with TCF- $\beta$ (" $\beta$ ") with or without TCF- $\beta R$ I inhibitor ("i"). Smad2/3 phosphorylation was measured by flow cytometry. Numbers indicate MFI of pSmad2/3. Data are representative of 3 independent experiments. Statistical analysis was performed by 2 -tailed $t$ test; ${ }^{*} P<0.05,{ }^{*} P<0.01,{ }^{* * *} P<0.001$. Error bars represent SD.

ratio with mixed bone marrow cells from B cell-deficient $\mu \mathrm{MT}$ mice and tamoxifen-treated $\operatorname{Ros} a 26^{\text {cre }} L$ rrc32 fff or Rosa26 $6^{W T} L r r c 32^{f / f}$ mice. In this model, all B cells were either GARP WT or KO, and at least $50 \%$ of all the remaining immune cells were WT. Stable B cell GARP KO was confirmed 3 months after bone marrow reconstitution in 72-hour LPS-treated peripheral B cells (Figure 3B).

The mixed BM chimeric mice were assessed at 6 months after bone marrow reconstitution, and strikingly we found evidence of spontaneous autoimmunity in BM chimera GARP-KO mice, including 
A

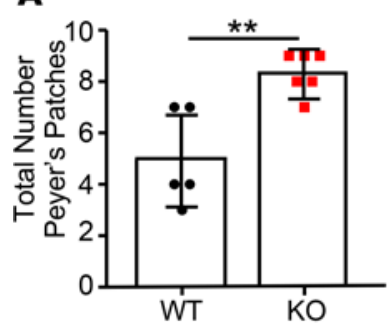

D

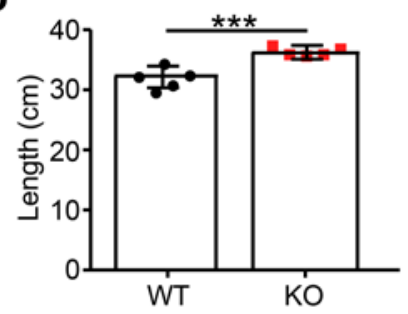

B

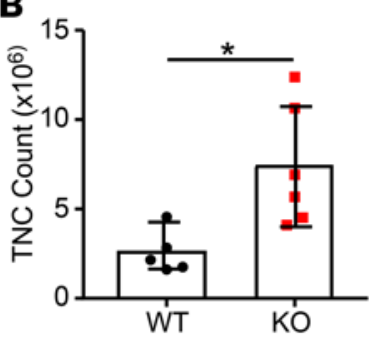

E

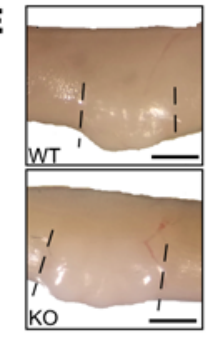

C

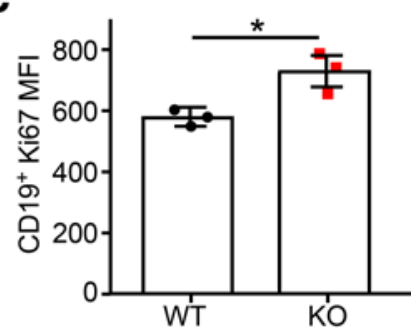

$\mathbf{F}$

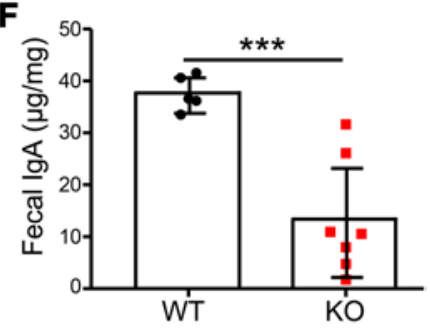

G
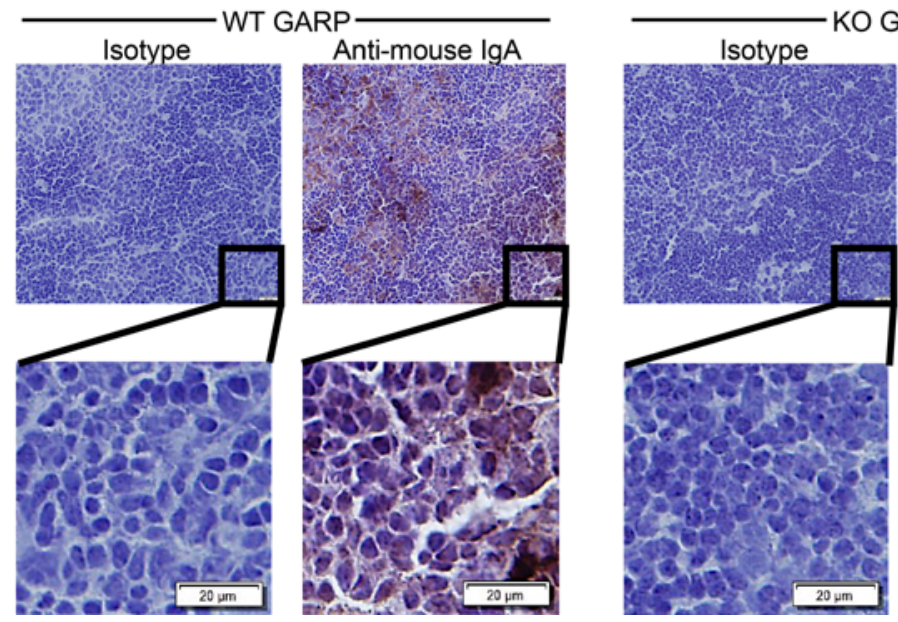

Anti-mouse IgA

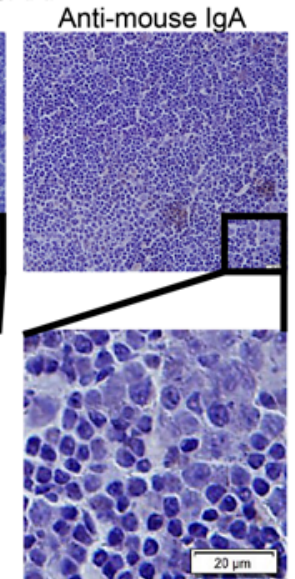

Figure 6. B cell-specific Lrrc32-- mice have Peyer's patch B cell hyperplasia and reduced mucosal IgA. The Peyer's patches were analyzed in 6-month-old BM chimera mice. (A) Differences in the total number of Peyer's patches between age-matched 6-month-old female WT and KO BMC mice ( $n=5$ WT and $n$ $=6 \mathrm{KO}$ biological replicates representative of 2 independent experiments). (B) Differences in cellularity of Peyer's patches between WT and KO BMC mice ( $n$ $=5 \mathrm{WT}$ and $n=6 \mathrm{KO}$ biological replicates representative of 2 independent experiments). TNC, total nucleated cells. (C) Flow cytometry quantification of Ki67 expression in CD19+ cells from Peyer's patches of WT and KO mice 6 months after bone marrow reconstitution ( $n=3$ replicates). Representative of 3 independent experiments. (D) Quantification of total small intestine length (duodenum to ileum) in WT and KO mice 6 months after bone marrow reconstitution ( $n=5$ WT and $n=6$ KO biological replicates). Representative of 2 independent experiments. (E) Representative images of size differences between Peyer's patches of GARP WT and KO mice 6 months after bone marrow reconstitution $(n=5)$. Scale bar: $0.5 \mathrm{~mm}$. (F) Fecal IgA levels from WT and GARP-KO BM chimera mice 6 months after bone marrow reconstitution were measured by ELISA ( $n=5$ WT and $n=7 \mathrm{KO}$ biological replicates). (C) IgA IHC on fresh frozen Peyer's patch sections from WT and KO BM chimera mice. Representative images from $n=3$ biological replicates. Scale bar: $20 \mu \mathrm{m}$. Statistical analysis was performed by 2 -tailed $t$ test; ${ }^{*} P<0.05,{ }^{* *} P<0.01,{ }^{* * *} P<0.001$. Error bars represent SD.

increased ANA (Figure 3, C and D), IgM and IgG deposition in the glomeruli of the kidney (Figure 3, E and $\mathrm{F}$ ), and hypergammaglobulinemia (Figure $3, \mathrm{G}$ and $\mathrm{H}$ ). We also measured the bacteria-specific IgA levels in the serum as an index of gut permeability due to loss of gut tolerance (48-50). While there was a systemic decrease in total IgA (Figure 3I), we observed that B cell-specific GARP-KO mice had a significant increase in IgA level against gut bacteria (Figure 3J).

Analysis of secondary immune compartments showed no difference in $\mathrm{CD}_{1} 9^{+}$cell percentage in the spleen; however, mesenteric lymph nodes ( $\mathrm{mLNs}$ ) of the KO mice had a significant increase of $\mathrm{CD} 19^{+}$cells (Figure 3K). An increase in highly proliferative B cells, as identified by intracellular Ki67, was observed in the mLN and small intestine (Supplemental Figure 1C; supplemental material available online with this article; https://doi.org/10.1172/jci.insight.99863DS1). The observation that B cell percentage and $\mathrm{Ki} 67^{+} \mathrm{B}$ cells were increased in the mucosal compartment of the GARP KO mice infers 
A

$\begin{array}{ccc}\text { Day 0 } & \text { Day 7 } & \text { Day 21 } \\ \text { OVA or } & \text { OVA + } & \text { Sacrifice } \\ \text { PBS gavage } & \text { CFA s.c. } & \end{array}$
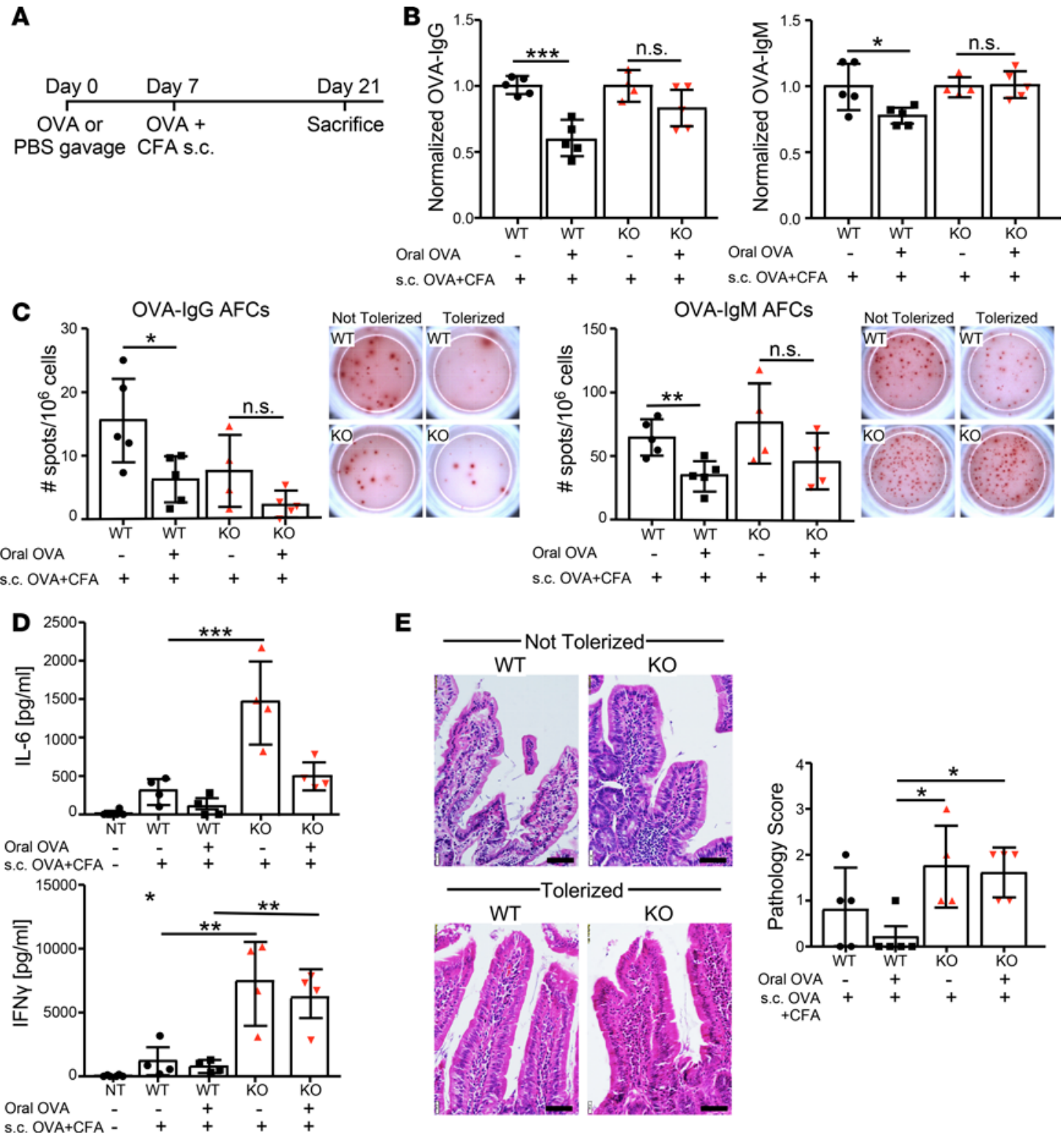

E

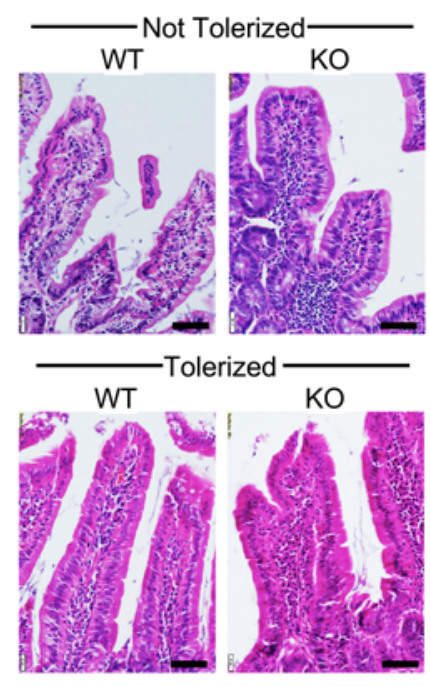

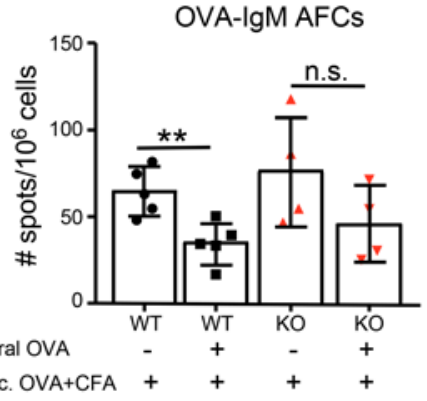

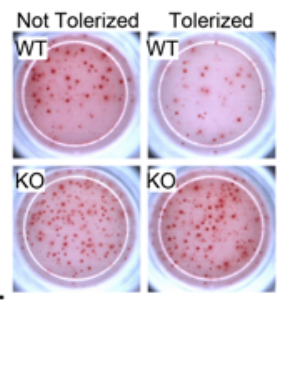

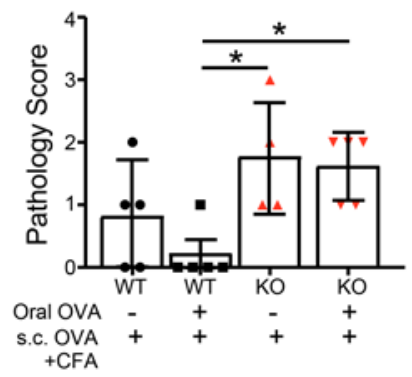

Figure 7. B cell-intrinsic GARP is indispensable for oral tolerance. (A) Schematic of oral tolerance model. Mice were given 25 mg OVA via gavage (tolerized) or PBS (not tolerized) as control, after which all mice received $100 \mu \mathrm{g}$ OVA emulsified in $100 \mu \mathrm{l}$ CFA s.c. Endpoint analysis occurred on day 21 , when mice were sacrificed. (B) OVA-specific IgG and IgM were detected in the sera of mice by ELISA ( $n=4-5$ biological replicates). (C) Splenic mononuclear cells were obtained from mice by mechanical separation and then cultured in OVA-coated ELISPOT plates for 20 hours, followed by detection with anti-mouse IgG1 and IgM. Quantitative data and representative well images are shown. (D) CD4+ $T$ cells were isolated by magnetic bead-positive selection from the spleens of WT and KO tolerized and nontolerized mice. CD4+ T cells were cultured in a 1:1 ratio with irradiated WT naive splenocytes in the presence of OVA for 4 days. No treatment (NT) indicates CD4+ cells cultured without OVA. Supernatants were collected, and IL- 6 and IFN- $\gamma$ levels were measured by ELISA. (E) Proximal (duodenum) small intestine tissues were fixed and sectioned, followed by H\&E staining. Representative images show duodenum villi. Graphical analysis of pathology score analyzed by a pathologist blinded to the treatment is shown. Statistical analysis was performed by 2-tailed $t$ test (B and $\mathbf{C}$ ) and 1-way ANOVA with Tukey's multiple comparisons ( $\mathbf{D}$ and $\mathbf{E}$ ); ${ }^{*} P<0.05$, ${ }^{* *} P<0.01,{ }^{* *} P<0.001$. Error bars represent SD.

in vivo B cell expansion, which strongly suggests that B cell-intrinsic GARP is an important negative checkpoint for B cell proliferation. Furthermore, we observed an increase in splenic myeloid cells (Supplemental Figure 1A) as well as Tregs in the spleen, mLN, PP, and small intestine lamina propria (Supplemental Figure 1B) in BM chimera GARP-KO mice, potentially reflecting a compensatory mechanism to control autoimmunity in response to B cell hyperactivation $(51,52)$. Despite the alterations in immune cells, there was no difference in systemic level of active or total TGF- $\beta 1$ (Supplemental Figure 1D). We concluded that deletion of Lrrc32 from B cells in mice leads to spontaneous lupus-like disease, despite the compensatory increase in Tregs. 
$B M$ chimera GARP-KO mice have increased susceptibility to chemical-induced experimental lupus. Multiple spontaneous and genetic mouse models of systemic lupus erythematosus (SLE) are commonly utilized to probe the pathogenesis of systemic autoimmune diseases (53). We first investigated whether GARP is aberrantly expressed on immune cells in the lupus-prone NZM2410 mouse model. Similar to human studies, prior experiments in lupus-prone mice have demonstrated reduced TGF- $\beta$ levels, predisposing the system to immune dysregulation and autoantibody production $(54,55)$. We found that GARP was significantly elevated on $\mathrm{CD} 19^{+} \mathrm{B}$ cells in the splenic compartment of 24-week-old NZM2410 mice with lupus but not young healthy mice (Supplemental Figure 2A). Further probing revealed that the marginal zone B cell population expressed GARP most highly, while transitional (CD21-CD23-) B cells had no GARP and LAP expression (Supplemental Figure 2B).

We next challenged the mixed BM chimera WT and GARP-KO mice with pristane to induce experimental lupus. The pristane-induced lupus (PIL) model recapitulates human SLE, as its pathogenesis is conferred by TLR hyperactivation and an enhanced type I IFN signature (56). WT or B cell-selective GARP-KO BM chimera mice were injected with a single dose of pristane i.p. 3 months after bone marrow reconstitution. We observed that GARP-KO mice developed IgG ANAs that remained elevated at 3 months as early as 2 weeks after pristane injection (Figure 4A). Other hallmarks of autoimmunity, such as proteinuria (Figure 4B), IgG (Figure 4C), and IgM kidney deposition (Figure 4D), were significantly more apparent in KO mice compared with WT mice. BM chimeric mice, on a C57BL6/J background, do not develop anti-dsDNA Ig or anti-chromatin IgG in response to pristane injection (57). Similar to observations in SLE patients $(58,59)$, active TGF- $\beta 1$, not total TGF- $\beta 1$, was significantly reduced in GARP$\mathrm{KO}$ mice with increased severity of disease (Figure $4 \mathrm{E}$ ). Interestingly, we observed elevated B cell GARP expression in WT mice with PIL, compared with minimal GARP expression at baseline prior to pristane treatment (Figure 4F). We also found that BM chimera GARP-KO mice had increased pulmonary hemorrhage and immune cell infiltration compared with WT mice (Figure 4G), in agreement with the notion that C57BL6/J mice are prone to pulmonary alveolar hemorrhage in the PIL model (57).

In an effort to confirm the integral nature of B cell-specific GARP in dampening early autoimmune induction, we crossed $h C D 20-E R^{c r e}$ transgenic mice (60) with $L r r c 32^{f / f}$ mice to generate $h C D 20-E R^{\text {cre }}$ Lrrc32 $2^{f / f}$ mice. Mice were treated with tamoxifen to delete GARP in B cells followed by injection with pristine to induce lupus (Supplemental Figure 3, A and B). We found that $h C D 20-E R^{\text {cre }} \operatorname{Lrc} 32^{f / f} \mathrm{~B}$ cell-specific GARP$\mathrm{KO}$ mice had a significant increase in ANA as early as 2 weeks after pristane injection compared with WT mice (Supplemental Figure 3, C and D). Taken together, we demonstrated that B cell-specific deletion of GARP promotes both spontaneous and chemical-induced lupus in mice and that GARP expression is induced on B cells in lupus-prone mice.

If GARP is a peripheral checkpoint for preventing autoimmune disease, we postulated that GARP OE would curtail PIL in mice. Indeed, we found that systemic OE of GARP reduced IgG ANA at 1 month and 4 months after pristane injection (Supplemental Figure 4A). There was also modest reduction in IgG glomerular deposition in GARP OE mice compared with WT mice (Supplemental Figure 4B). While GARP OE was not limited to the B cell compartment in this model, B cells were the most altered at the 4-month endpoint analysis. In the spleen, the percentage of germinal center B cells $\left(\mathrm{GL} 7^{+} \mathrm{CD} 19^{+}\right)$was significantly reduced as well as the percentage of $\mathrm{Ki}^{+} 7^{+} \mathrm{B}$ cells (Supplemental Figure 4C). The percentage of $\mathrm{Gr}^{+} \mathrm{CD} 11 \mathrm{~b}^{+}$granulocytic cells was also significantly decreased in GARP OE mice (Supplemental Figure 4D), as opposed to the increase in granulocytic cells seen in B cell-specific GARP-KO BM chimeric mice (Supplemental Figure 1A). In the PPs and mLNs (Supplemental Figure 4E), there were alterations in CD4 ${ }^{+}$ and $\mathrm{CD}^{+} \mathrm{T}$ cells compartments. Active TGF- $\beta 1$ was significantly reduced in both GARP WT and OE mice after pristane treatment, although GARP OE mice had a significant increase in total TGF- $\beta 1$ pre-PIL (Supplemental Figure 4F). Strikingly, GARP OE mice had an increase in soluble GARP in the serum after pristane treatment (Supplemental Figure 4G), which has been implicated in promoting tolerance $(13,34)$. Thus, the roles of GARP-LTGF- $\beta 1$ in immune tolerance and systemic autoimmunity are illustrated by both loss-of-function and gain-of-function studies.

PP B cells regulate peripheral tolerance via increased responsiveness to the GARP-LTGF- $\beta$ axis. B cells $(61,62)$, as well as PPs (63), are required for mucosal tolerance to both self and oral antigens. We observed that GARP OE B cells from the PP had elevated pSmad2/3 (Figure 2G), and B cell-specific GARP-KO mice had a significant increase in total B cells as well as $\mathrm{Ki}^{+} 7^{+} \mathrm{B}$ cells in the $\mathrm{mLNs}$ but not in the spleen (Figure $3 \mathrm{~K}$ and Supplemental Figure 1C). These data raised an intriguing possibility that the GARP-LTGF- $\beta$ axis 
plays strategically important roles for mucosal tolerance. In agreement with this hypothesis, we found that WT PP B cells expressed elevated levels of Lrrc32 transcript at baseline, compared with splenic WT B cells (Figure 5A). Consistent with the increase in Lrrc32 expression level, PP B cells responded to both BCR ligation and TLR stimulation by upregulating GARP at an elevated level compared with splenic B cells (Figure 5B). At 48 hours after stimulation, anti- $\mu$ antibody and LPS-treated GARP-KO PP B cells had increased proliferation compared with WT cells (Figure $5 \mathrm{C}$ ), which is in line with the finding that GARP OE B cells have reduced proliferation. The finding that IgA secretion from 96-hour LPS-treated PP B cells was reduced in GARP-KO cells (Figure 5D) is also consistent with reduced autocrine TGF- $\beta$ signaling.

Mucosal tissues are highly enriched with active TGF- $\beta$ and associated cofactors (e.g., retinoic acid, BAFF, and APRIL), which explains why IgA CSR in the gut-associated lymphoid tissues is more pronounced compared with other secondary lymphoid organs (64). Importantly, gut B cells produce TGF- $\beta$ in an autocrine fashion $(65,66)$. Phenotypic analysis revealed baseline differences between WT splenic and PP B cells, including increased CD1d, MHC class I, MHC class II, CD40, and CD86 expression in PP B cells (Figure 5E), indicating that the $\mathrm{B}$ cells in the $\mathrm{PP}$ compartment are more poised for responses to antigens than splenic B cells. In order to link the alterations in proliferation and IgA CSR in PP B cells to GARPTGF- $\beta$ axis, we assessed pSmad2/3 levels in WT versus GARP KO B cells treated with anti- $\mu$ antibody or LPS for 96 hours, which induced high endogenous GARP expression on the WT cells. Ex vivo analysis of WT and KO splenic and PP B cells revealed that GARP-deficient PP B cells exhibited a significant reduction in pSmad2/3 signaling (Figure 5, F and G), whereas splenic B cells did not have this GARP-driven difference. PP WT and GARP-KO B cells responded equally to exogenous TGF- $\beta$ (Figure $5 \mathrm{H}$ ), indicating that TGF- $\beta$ bioavailability, not the TGF- $\beta$ signaling machinery, is effected by GARP expression.

The profound effect of cell surface of GARP-TGF- $\beta$ on PP B cell biology was further revealed in vivo in BM chimera GARP-KO mice. Deletion of GARP from B cells resulted in a significantly increased number of PPs in mice (Figure 6A). Higher number of PPs also correlated with increased PP cellularity (Figure $6 \mathrm{~B})$ and elevated $\mathrm{CD} 19^{+} \mathrm{B}$ cell proliferation, as measured by Ki67 $7^{+}$staining 6 months after bone marrow reconstitution (Figure 6C). Conversely, GARP OE reduced the total number of PPs (Supplemental Figure $5 \mathrm{~A}$ ), percentage of B cells (Supplemental Figure 5B), and B cell Ki67+ expression level (Supplemental Figure 5 C). As further evidence of loss of B cell homeostasis, the BM chimera GARP-KO mice were observed to have increased small intestinal length (Figure 6D) as well as grossly larger PPs (Figure 6E). Fecal IgA levels (Figure 6F) and the number of IgA-producing cells in the PPs (Figure 6G) were notably reduced in GARP-KO mice compared with WT mice. The heightened responsiveness of PP B cells to TGF- $\beta$ is explained by a significant increase in TGF- $\beta$ RII expression compared with splenic B cells (Supplemental Figure 5D). Notably, while there is no difference in TGF- $\beta$ RII mRNA level between WT and OE B cells, GARP OE B cells do have a significant increase in $\mathrm{p} 21$, which is a downstream target in the TGF- $\beta$ signaling pathway (Supplemental Figure 5D).

$B$ cell-intrinsic GARP is indispensable for oral tolerance. We next addressed the role of B cell-intrinsic GARP in oral tolerance for several reasons: first, PP B cells appear to be more sensitive to regulation by GARP; second, PPs are required for oral tolerance (67); and third, the GARP-TGF- $\beta$ axis on B cells is required for maintaining systemic tolerance. To this end, we utilized a chick ovalbumin (OVA) oral tolerance model (Figure 7A) $(68,69)$ to determine if oral tolerance is effected by $\mathrm{GARP}^{+} \mathrm{B}$ cells. While BM chimera WT mice were tolerized against high-dose oral OVA prior to immunization, BM chimera GARP-KO mice did not have a significant decrease in mounting systemic OVA-specific antibody response, measured by anti-OVA antibody (Figure 7B), and OVA-specific antibody forming cells (AFCs) (Figure 7C). Consistent with OVA being a T cell-dependent antigen, we found that B cell-specific GARP-KO mice mount a much-increased antigen-specific $\mathrm{T}$ cell response. Importantly, prior administration of antigen orally failed to tolerize the elevated $\mathrm{T}$ cell responses in the KO mice (Figure 7D). Pathological analysis of the proximal small intestine further demonstrated evidence for loss of tolerance in the BM chimera GARP-KO mice (Figure 7E). We conclude that B cell-intrinsic GARP plays important roles in oral tolerance.

\section{Discussion}

TGF- $\beta$ is a pleiotropic cytokine with critical roles in immune cell development, homeostasis, and tolerance (20). However, there remain many unanswered questions, particularly in the context of regulation via GARP. Our study deals with cell surface TGF- $\beta$ in peripheral immune tolerance, which has eluded the field for so long due to lack of understanding of the molecular basis of its cell surface expression. 
Recently, the expression of a cell surface docking receptor for TGF- $\beta$, GARP, has been described on Tregs $(3,4,10)$, platelets $(4,7)$, and cancer cells $(13)$. While probing the roles of GARP in immunity, we discovered that GARP is significantly upregulated on peripheral B cells in systemic autoimmune diseases and GARP expression is the basis for TGF- $\beta$ presence on the cell surface. Importantly, we found that multiple MyD88-dependent TLR ligands (70), including ligands for TLR4, TLR7, and TLR9, drive surface GARP expression on normal B cells, raising an intriguing possibility that the GARP-TGF- $\beta$ axis on activated B cells is a checkpoint for maintaining B cell peripheral tolerance. We addressed this hypothesis critically using multiple genetic models, including GARP conditional $\mathrm{KO}$ and OE systems. We conclusively demonstrated that (a) loss of GARP from B cells promotes spontaneous and chemical-induced lupus-like disease in mice; (b) gain of GARP dampens $\mathrm{T}$ cell-independent antibody production and $\mathrm{B}$ cell proliferation, while promoting IgA CSR; (c) PP B cells are especially dependent on the GARP-TGF- $\beta$ axis to regulate proliferation, IgA production, and thus, tolerance; and (d) GARP on B cells plays indispensable roles in oral tolerance of $\mathrm{T}$ cell-dependent antigens.

Mechanistically, there are multiple possible reasons why the B cell-intrinsic GARP-TGF- $\beta$ axis is important for peripheral B cell tolerance. First, GARP expression induced by TLR ligands ensures that $B$ cell activation does not get out of control via the autocrine antiproliferative effect of TGF- $\beta$. Second, GARP promotes IgA CSR via enhancing TGF- $\beta$ activity, which has been suggested by a recent in vitro study (44), and is now conclusively revealed by our study of mucosal IgA production in vivo in the context of autoimmune diseases. Mucosal IgA production plays important roles in mucosal defense against pathogens or commensal bacteria that fortuitously cross the gut epithelium barriers (71-73). Third, GARP expression could also inhibit the ability of TLR-activated B cells to produce proinflammatory cytokines via a TGF- $\beta$-dependent mechanism, an area that is being actively studied in our laboratory.

One of the most striking findings of our work is that PP B cells are more dependent on the GARPTGF- $\beta$ axis compared with splenic B cells for homeostasis. Without GARP, PP B cells undergo enhanced proliferation and activation, but the ability of these B cells to differentiate into IgA-producing cells is compromised. These data suggest that PPs are critical organs for mucosal B cell tolerance. PP lymphoid organs are in a precarious position, as they are constantly exposed to varying levels of TLR agonists via the gut microbiome (74). It makes teleological sense for PP B cells therefore to be equipped with more robust peripheral tolerance mechanisms, such as the GARP-TGF- $\beta$ axis. A recent comprehensive study of the B cell clonal distribution in humans revealed two broad clonal networks: one encompassing the blood, bone marrow, spleen, and lungs, and the other that is restricted to tissues within the gastrointestinal tract (75). The group observed that the GI tract clones have higher frequencies of somatic hypermutation, which suggests a biological relevance for the heightened importance for GARP in the PP immune compartment in order to tolerize self and oral antigens. Further evidence of this argument is found in a 2016 GWAS study: the C11orf30-LRRC32 locus is associated with multiple "immune-related phenotypes," such as inflammatory bowel diseases and allergy (76-78).

There is an increased expression of GARP on B cells in murine models of lupus. Based on the tolerogenic roles of B cell GARP discovered in our study, we believe that GARP upregulation in autoimmune setting represents a compensatory mechanism to return B cells to normal homeostasis. This is perfectly consistent with the fact that TLR signaling is prevalent in lupus $(79,80)$ but also induces GARP expression. This reasoning begs for two important future lines of investigations: first, are there any SLE patients who are unable to upregulate GARP and thus develop worse clinical diseases? Second, can biologically active soluble GARP be used therapeutically for autoimmune diseases? In murine cancer models, blocking GARP via antibody has been demonstrated to be beneficial $(13,36)$. Due to the multifactorial functions of TGF- $\beta$, targeting TGF- $\beta$ directly in the clinic is challenging (81). A GARP-based therapeutic strategy may be a more feasible method of treating autoimmunity in the clinic; however, caution is warranted, as it is necessary to strike a balance between cancer immune surveillance and immune tolerance.

Despite a battery of intriguing findings in the study, many questions still remain in the context of $B$ cell GARP and tolerance. NF- $\mathrm{kB}$ is the common downstream molecule of multiple MyD88-dependent TLR signaling cascades (70). It is possible that TLR signaling induces GARP expression via NF- $\mathrm{BB}$, as suggested by the presence of a putative NF- $\mathrm{BB}$-binding site in the promoter region of Lrrc32 (82). However, further study is necessary to clarify how GARP is regulated by TLR pathways. GARP is known to bind to TGF- $\beta 1$, TGF- $\beta 2$, and TGF- $\beta 3$ (45). There is evidence that resting B cells express higher levels of TGF- $\beta 3$ than activated B cells and are able to expand Treg populations in a TGF- $\beta 3$-dependent 
mechanism (83). However, GARP is not expressed by resting B cells, which leads us to believe that GARP primarily regulates the bioavailability of TGF- $\beta 1$ and TGF- $\beta 2$ isoforms, rather than TGF- $\beta 3$, to exert its regulatory roles in $\mathrm{B}$ cells. In addition, this work focused on peripheral tolerance, not central $\mathrm{B}$ cell tolerance, primarily because GARP was not found on immature B cells in the bone marrow. However, given the central roles of TGF- $\beta$ in the negative selection of B cells in the bone marrow (84), the roles of GARP in the process cannot be completely ruled out. Finally, this study focused on the B cell-intrinsic role of GARP in regulating tolerance; however, B cells may also regulate tolerance via interaction with T cells. For example, LPS-primed B cells are less potent stimulators of CD8 ${ }^{+} \mathrm{T}$ cells than anti-Ig/ anti-CD40-stimulated B cells, which also illustrates a TGF- $\beta 1-$ dependent mechanism (85).

This is the first study to our knowledge demonstrating that the cell surface GARP-TGF- $\beta$ axis modulates systemic tolerance in a B cell-intrinsic manner via autocrine TGF- $\beta$ signaling. We reached our conclusion through several genetic strategies. We anticipate that this study will be a launching point for further investigations into the role of GARP in the pathogenesis of human autoimmune diseases as well as the possibility of targeting GARP as a novel diagnostic and therapeutic strategy for autoimmune diseases.

\section{Methods}

Mice. We developed an inducible GARP OE mouse model using the "Flexible Accelerated STOP TetO knockin system" (Ingenious Targeting Laboratory) (86). Development of the GARP-FAST mouse model has been described previously (45). GARP OE was induced with doxycycline (50 $\mu \mathrm{g} / \mathrm{ml}$; MilliporeSigma) in 1\% sucrose drinking water. Lrrc32 ffoxffox mice were obtained from Riken (9) and crossed with Rosa26-ER ${ }^{\text {cre }}$ mice (The Jackson Laboratory) to generate tamoxifen-induced total-body GARP-KO mice. $h C D 20-E R^{c r e} L r r c 32^{f / f}$ mice were generated by crossing $h C D 20-E R^{c r e}$ mice, which were previously described (60), with $L r r c 32^{f f f}$ mice. Induction of GARP KO in both models was achieved with i.p. injection of tamoxifen (MilliporeSigma) dissolved in peanut oil. Mice received a dose of $5 \mu \mathrm{g} / \mathrm{g}$ body weight daily for 7 days. C57BL6/J and $\mu \mathrm{MT}$ mice were purchased from The Jackson Laboratory. NZM2410 mice were a gift from Gary Gilkeson (Medical University of South Carolina [MUSC]). B cell-specific bone marrow chimera mice were generated by lethally irradiating (2 doses of 550 Gy, 6 hours apart) female CD45.1 C57BL6/J mice. Twenty-four hours after lethal irradiation, mice were i.v. injected with $2 \times 10^{6}$ bone marrow cells from $\mu \mathrm{MT}$ and tamoxifen-treated Rosa $26^{\text {cre }}$ Lrrc $32^{f / f}$ or Rosa26 $6^{W T}$ Lrrc $32^{f / f}$ mice mixed in a 1:1 ratio. All mice, except the NZM2410 strain, were on a pure C57BL6/J background, and were age matched for experiments. Unless otherwise stated, mice were between 6 weeks and 6 months of age for experiments. Female mice were used for all lupus and tolerance experiments, due to the low rate of autoimmunity observed in male mice.

Human samples. Deidentified whole-blood samples from healthy controls were received from the MUSC Multidisciplinary Clinical Research Center in heparin-coated tubes. Peripheral blood lymphocytes were isolated using a Ficoll-Paque PLUS gradient (GE Healthcare).

Cell culture. To obtain purified mouse B cells, spleens and PPs were aseptically isolated and mashed through a $40-\mu \mathrm{M}$ filter to obtain a single-cell suspension and treated with ACK buffer (MilliporeSigma) to lyse RBC. Mouse B cells were isolated from splenocytes or PP lymphocytes using mouse CD19+ magnetic beads following the manufacturer's protocol (Miltenyi Biotec). Human CD19+ magnetic beads (Miltenyi Biotec) were used to isolate B cells from human PBMCs. In all experiments, cells were cultured in RPMI (Corning) supplemented with 10\% FBS (Atlas Biologicals), 1\% penicillin/ streptomycin (ThermoFisher), and 0.1\% 2-mercaptoethanol (MilliporeSigma). Mouse B cells were treated with anti- $\mu(5 \mu \mathrm{g} / \mathrm{ml}$, unless otherwise noted; Jackson ImmunoResearch), LPS (10 $\mu \mathrm{g} / \mathrm{ml} ; E$. coli 055:B5, MilliporeSigma), R848 (2.5 $\mu \mathrm{g} / \mathrm{ml}$; InvivoGen), or CpG (1 $\mu \mathrm{M}, \mathrm{BW}-006$ InvivoGen). In experiments with exogenous TGF- $\beta$ treatment and TGF- $\beta$ RI inhibition, cells were treated for 1 hour with TGF- $\beta$ RI inhibitor dissolved in DMSO (SB 431542 hydrate, MilliporeSigma) prior to the addition of $2 \mathrm{ng} / \mathrm{ml}$ TGF $\beta 1$ (PeproTech).

Lupus induction and autoimmune analysis. Female BM chimera or doxycycline-treated GARP OE mice were given a single i.p. injection of pristane (500 $\mu 1 / 20 \mathrm{~g}$ mouse; MilliporeSigma). GARP OE and WT littermates were fed doxycycline $(50 \mu \mathrm{g} / \mathrm{ml})$ in the drinking water throughout the course of the experiment.

$A N A$. ANAs were detected using NOVA Lite Hep-2 slides (Inova Diagnostics) and FITC-conjugated anti-mouse IgG or anti-mouse IgM antibody (ThermoFisher). Serum was diluted at the stated ratios in PBS. Fluorescent intensity was analyzed in a blinded manner and recorded on a scale of $0-4$ on a Zeiss microscope, as described previously (87). 
Immune deposition. Immune deposition in the kidneys was analyzed using fresh frozen kidney sections embedded in Cryomatrix solution (ThermoFisher). Slides were fixed in ice-cold acetone and then blocked overnight using the M.O.M. kit (Vector Labs). Slides were stained with either IgM-FITC or IgG-FITC antibodies (ThermoFisher) for 30 minutes at room temperature and then coverslipped with anti-fade mounting medium (Southern Biotech). The fluorescent intensity of 25 nonoverlapping glomeruli was averaged to obtain the kidney deposition score as described before (88).

Urine protein. Urine was collected from the mice at the 3-month endpoint. Protein concentration was measured with a Bradford assay (Bio-Rad) using 1:50 urine diluted in PBS and a BSA standard curve.

Histology. Mouse lungs were perfused with $10 \%$ formalin prior to resection and fixed in $4 \%$ formalin. Fixed lungs were embedded in paraffin, sectioned, and stained following a standard H\&E staining protocol.

Flow cytometry. Spleens, mLNs, and PPs were dissociated into a single-cell suspension, and splenocytes were depleted of RBCs with RBC lysis buffer (MilliporeSigma). After Fc-receptor blocking, cells were stained for surface markers in FACS buffer. Antibodies against mouse CD4 (clone RM4-5), FoxP3 (FJK-16), CD11b (M1/70), GARP (YGIC86), LAP (TW7-16B4), IgD (11-26), IgM (eb121-15F9), CD23 (B3B4), CD86 (GL1), MHC-I (SF-1.1.1), MHC-II (M5/114.15.2), CD44 (IM7), CD62L (MEL-14), PD-L1 (B7-H1), CD1d (L363), Helios (22F6), and CD5 (53-7.3) were purchased from ThermoFisher. Anti-mouse CD8a (53-6.7), Gr1 (RB6-8C5), CD21 (7G6), CD80 (16-10A1), PD-1 (J43), Ki67 (SolA15), and pSmad (O72-670) were purchased from BD Biosciences. Anti-mouse B220 (RA3-6B2), CD19 (6D5), and GL7 were purchased from BioLegend. All intracellular staining was performed using the commercial FoxP3/ Transcription Factor Staining Buffer Set (Affymetrix). Analysis of pSmad2/3 was performed using BD Phosflow buffer sets according to the manufacturer's protocol (BD). Samples were analyzed on a BD LSR cytometer and analyzed by FlowJo Software (Tree Star). Viability of the cells analyzed was ensured by gating on singlets and live cells, as indicated by the lack of 7-AAD or Fixable Viability Dye (Affymetrix).

TNP-ficoll immunization. Doxycycline-treated WT and GARP OE mice were bled at baseline via facial vein to collect serum and immunized with TNP-AECM-Ficoll $(50 \mu \mathrm{g} / \mathrm{ml}$ in PBS; Biosearch Technologies) injected in the peritoneal cavity. Mice were bled weekly for 5 weeks. TNP-ficoll-specific IgM antibody levels were detected by ELISA. ELISA plates (Corning) were coated overnight at $4^{\circ} \mathrm{C}$ with TNP-ficoll (50 $\mu \mathrm{g} / \mathrm{ml}$ in PBS), followed by blocking with $1 \%$ BSA for 3 hours. Diluted serum was incubated for 1 hour at room temperature while shaking; TNP-specific IgM antibodies were detected with anti-mouse IgM-HRP (Southern Biotech).

Oral tolerance model. Five-month-old reconstituted female bone marrow chimera WT and GARP-KO mice were fed either $25 \mathrm{mg}$ OVA peptide (MilliporeSigma) or PBS as control via oral gavage. Seven days later, both OVA- and PBS-treated mice were given $100 \mu \mathrm{g}$ OVA peptide (BioVendor) emulsified in $100 \mu \mathrm{l}$ Complete Freund's Adjuvant (InvivoGen) s.c. Mice were sacrificed 14 days after s.c. immunization. Serum was collected for OVA-specific IgM and IgG ELISA as well as splenocytes for ELISPOT and OVA-induced $\mathrm{CD}^{+} \mathrm{T}$ cell cytokine production assay.

ELISPOT. Spleens from tolerized (OVA gavage and OVA-CFA s.c. immunization) and not tolerized (PBS gavage and OVA-CFA s.c. immunization) WT and GARP-KO BM chimera mice were mashed into a single-cell suspension. Cells were plated at $1 \times 10^{6}$ cells/well in High-protein binding Immobilon-P (Millipore) plates that were activated with $30 \%$ ethanol and precoated overnight with $100 \mu \mathrm{g} / \mathrm{ml}$ OVA. Splenocytes from each mouse were cultured in triplicate and incubated for 20 hours at $37^{\circ} \mathrm{C}$. Plates were washed 3 times with PBS followed by 3 washes with PBS with $0.5 \%$ Tween-20; then, either anti-mouse IgG1-HRP or anti-mouse IgM-HRP detections antibodies were added for 2 hours. After further washes, AECM substrate (MilliporeSigma) was added and the reaction was stopped with distilled water after color development. Images and quantification were performed using the AID EliSpot Reader System ELR04 (Autoimmun Diagnostika $\mathrm{GmbH})$.

OVA-induced $\mathrm{CD}^{+} \mathrm{T}$ cell cytokine production assay. $\mathrm{CD}^{+} \mathrm{T}$ cells were isolated from RBC-depleted splenocytes using anti-mouse CD4 ${ }^{+}$MACS beads (Miltenyi Biotech). Purified CD4 ${ }^{+} \mathrm{T}$ cells from tolerized and not tolerized WT and GARP-KO mice were cultured in a 1:1 ratio with WT irradiated untreated splenocytes, which provided a source of antigen-presenting cells. $100 \mu \mathrm{g} / \mathrm{ml}$ OVA peptide was added to each well, and cells were incubated for 96 hours at $37^{\circ} \mathrm{C}$. Supernatants were collected and analyzed for IL- 6 and IFN- $\gamma$ by ELISA.

ELISA. To measure active and total TGF- $\beta 1$ in both cell culture supernatants and serum, ELISA plates (Corning) were coated overnight at $4^{\circ} \mathrm{C}$ with anti-mouse/human TGF- $\beta 1$ capture antibody (BioLegend), 
followed by blocking with $1 \%$ BSA for 1 hour at room temperature. Samples were treated with $\mathrm{HCl}$ for 10 minutes and neutralized with Tris/ $\mathrm{NaOH}$ to obtain total TGF- $\beta$. Both active and total TGF- $\beta 1$ were detected using anti-mouse Biotin-TGF- $\beta$ antibody (BioLegend), Streptavidin-HRP (BioLegend), and TMB Substrate reagents (BD). To detect soluble GARP in both mouse and human serum, a human/mouse GARP ELISA kit was used according to the manufacturer's protocol (BioLegend). Immunoglobulin levels were detected in both serum and cell culture supernatant using a mouse antibody Isotype kit (Southern Biotech). After overnight coating of the plates, plates were blocked with 1\% BSA for 3 hours followed by standard ELISA procedure described above. To determine the IgA level in feces, fecal pellets were collected and weighed. $10 \mu 1 \%$ BSA in PBS with protease inhibitor cocktail (MilliporeSigma) was added per $\mathrm{mg}$ feces and homogenized. Following centrifugation, fecal supernatant was added to the blocked ELISA plate at 1:1,000 dilution. To detect bacteria-specific IgA in the sera, a fecal supernatant from $\mu \mathrm{MT}$ mice was prepared in the method described above, and the protein concentration was measured using Bradford assay (Bio-Rad). Each well of the ELISA plate was coated with $0.5 \mu \mathrm{g} / \mathrm{ml} \mu \mathrm{MT}$ fecal supernatant. IL-6 and IFN- $\gamma$ ELISAs were performed according to manufacturer's protocol (BD).

$q R T-P C R$ and immunoblot. RNA was isolated from purified splenic and PP B cells using Trizol Reagent according to the manufacturer's protocol (Life Technologies). cDNA was made, following the manufacturer's instructions, with the iScript cDNA synthesis kit (Bio-Rad). Primer sequences were as follows: mouse GARP forward 5'-CGCTTCGTCACCTGGATTTA-3', mouse GARP reverse 5'-ATTGTGGGCCAGGTTAAGG-3', mouse $\beta$-actin forward 5'-AGCTGAGAGGGAAATCGTGC-3', and mouse $\beta$-actin reverse 5'-TCCAGGGAGGAAGAGGATGC-3'. qRT-PCR was run on a StepOne Plus machine (Applied BioSystems). For immunoblot analysis, cell lysate was made using RIPA buffer supplemented with protease inhibitor cocktail (MilliporeSigma). $75 \mu \mathrm{g}$ lysate was run on a 10\% SDS-PAGE. After transfer to a PVDF membrane (Millipore), GARP was probed using an anti-mouse GARP antibody (R\&D Systems) and normalized to $\beta$-actin (MilliporeSigma)

Immunohistochemistry. Small intestine sections with PPs were embedded in Cryomatrix freezing medium and snap frozen. Five- $\mu \mathrm{m}$ sections were cut and fixed with ice-cold acetone, followed by blocking and incubating with IgA-HRP (Southern Biotech). Color was detected using the DAB Peroxidase Developing Kit (Vector Laboratories). Images were acquired on an Olympus microscope.

Statistics. Data analysis was performed using GraphPad Prism software (GraphPad Software Inc.). Analyses were performed using unpaired 2-tailed $t$ test or Mann-Whitney for nonparametric data or ANOVA, as indicated. One-way ANOVA with Tukey's comparison was used for analyses with multiple comparisons. $P$ values of less than 0.05 were considered significant.

Study approval. Healthy controls provided informed consent for peripheral blood sample collection by the Multidisciplinary Clinical Research Center for Rheumatic Diseases at the MUSC. The MUSC IRB approved the protocols for these studies (Pro00021985 and Pro27985), and the project was conducted according to the Declaration of Helsinki. All animal procedures in this study were conducted following a protocol approved by the MUSC Institutional Animal Care and Use Committee. Animals were housed in specific pathogen-free conditions.

\section{Author contributions}

ZL supervised the project. CHW, BL, and ZL designed the experiments. $\mathrm{CHW}$ and BXW performed the experiments. SS did most of the histological analysis. GG facilitated the acquisition of clinical samples. MJS provided mice. All authors, including MS, EAAA, and AM, contributed to the data analysis and assisted with manuscript preparation.

\section{Acknowledgments}

This project was supported by multiple grants from the NIH: UL1TR001450 and TL1TR001451 (to CHW); R01AI077283 (to ZL); R01CA213290 (to ZL); R01CA188419 and P01CA186866 (to ZL); R01CA193939 (to BL); and U01AI125859 (to BL). It was also supported in part by the Cell Evaluation \& Therapy Shared Resource, the Hollings Cancer Center, MUSC (P30CA138313), and the Division of Rheumatology and Immunology at the Multidisciplinary Clinical Research Center (NIH/National Institute of Arthritis and Musculoskeletal and Skin Diseases) and P60AR062755 (to GG). We thank Wei Jiang (MUSC) for the use of the AID ELISPOT reader in her laboratory. The authors thank the past and present members of the $\mathrm{Li}$ laboratory for stimulating discussions throughout the course of the study. 
Address correspondence to: Zihai Li or Bei Liu, 86 Jonathan Lucas Street, Hollings Cancer Center, HO612B (Z. Li) or HO612H (B. Liu), Charleston, South Carolina 29425, USA. Phone: 843.792.1034; Email: zihai@musc.edu (Z. Li). Phone: 843.792.8994; Email: liube@musc.edu (B. Liu).

1. Ollendorff V, Noguchi T, deLapeyriere O, Birnbaum D. The GARP gene encodes a new member of the family of leucine-rich repeat-containing proteins. Cell Growth Differ. 1994;5(2):213-219.

2. Stockis J, Colau D, Coulie PG, Lucas S. Membrane protein GARP is a receptor for latent TGF-beta on the surface of activated human Treg. Eur J Immunol. 2009;39(12):3315-3322.

3. Wang R, Kozhaya L, Mercer F, Khaitan A, Fujii H, Unutmaz D. Expression of GARP selectively identifies activated human FOXP3+ regulatory T cells. Proc Natl Acad Sci USA. 2009;106(32):13439-13444.

4. Tran DQ, Andersson J, Wang R, Ramsey H, Unutmaz D, Shevach EM. GARP (LRRC32) is essential for the surface expression of latent TGF-beta on platelets and activated FOXP3+ regulatory T cells. Proc Natl Acad Sci USA. 2009;106(32):13445-13450.

5. Wang R, Zhu J, Dong X, Shi M, Lu C, Springer TA. GARP regulates the bioavailability and activation of TGF $\beta$. Mol Biol Cell. 2012;23(6):1129-1139.

6. Fridrich S, et al. How soluble GARP enhances TGF $\beta$ activation. PLoS One. 2016;11(4):e0153290.

7. Rachidi S, et al. Platelets subvert T cell immunity against cancer via GARP-TGFß axis. Sci Immunol. 2017;2(11):eaai7911.

8. Zhou AX, Kozhaya L, Fujii H, Unutmaz D. GARP-TGF- $\beta$ complexes negatively regulate regulatory T cell development and maintenance of peripheral CD4+ T cells in vivo. J Immunol. 2013;190(10):5057-5064.

9. Edwards JP, Fujii H, Zhou AX, Creemers J, Unutmaz D, Shevach EM. Regulation of the expression of GARP/latent TGF- $\beta 1$ complexes on mouse T cells and their role in regulatory T cell and Th17 differentiation. J Immunol. 2013;190(11):5506-5515.

10. Zhang Y, et al. GP96 is a GARP chaperone and controls regulatory T cell functions. J Clin Invest. 2015;125(2):859-869.

11. Li Y, et al. Hepatic stellate cells inhibit T cells through active TGF- $\beta 1$ from a cell surface-bound latent TGF- $\beta 1 / G A R P$ complex. J Immunol. 2015;195(6):2648-2656.

12. Carrillo-Galvez AB, et al. Mesenchymal stromal cells express GARP/LRRC32 on their surface: effects on their biology and immunomodulatory capacity. Stem Cells. 2015;33(1):183-195.

13. Metelli A, et al. Surface Expression of TGF $\beta$ docking receptor GARP promotes oncogenesis and immune tolerance in breast cancer. Cancer Res. 2016;76(24):7106-7117.

14. Hahn SA, et al. A key role of GARP in the immune suppressive tumor microenvironment. Oncotarget. 2016;7(28):42996-43009.

15. Jin H, Sun L, Tang L, Yu W, Li H. Expression of GARP is increased in tumor-infiltrating regulatory T cells and is correlated to clinicopathology of lung cancer patients. Front Immunol. 2017;8:138.

16. Shull MM, et al. Targeted disruption of the mouse transforming growth factor-beta 1 gene results in multifocal inflammatory disease. Nature. 1992;359(6397):693-699.

17. Yaswen L, et al. Autoimmune manifestations in the transforming growth factor-beta 1 knockout mouse. Blood. 1996;87(4):1439-1445.

18. Annes JP, Munger JS, Rifkin DB. Making sense of latent TGFbeta activation. J Cell Sci. 2003;116(Pt 2):217-224.

19. Feng XH, Derynck R. Specificity and versatility in tgf-beta signaling through Smads. Annu Rev Cell Dev Biol. 2005;21:659-693.

20. Li MO, Flavell RA. TGF-beta: a master of all T cell trades. Cell. 2008;134(3):392-404.

21. Munger JS, et al. The integrin alpha $\mathrm{v}$ beta 6 binds and activates latent TGF beta 1: a mechanism for regulating pulmonary inflammation and fibrosis. Cell. 1999;96(3):319-328.

22. Gentry LE, Nash BW. The pro domain of pre-pro-transforming growth factor beta 1 when independently expressed is a functional binding protein for the mature growth factor. Biochemistry. 1990;29(29):6851-6857.

23. Brown TL, Patil S, Howe PH. Analysis of TGF-beta-inducible apoptosis. Methods Mol Biol. 2000;142:149-167.

24. Wildey GM, Patil S, Howe PH. Smad3 potentiates transforming growth factor beta (TGFbeta )-induced apoptosis and expression of the BH3-only protein Bim in WEHI 231 B lymphocytes. J Biol Chem. 2003;278(20):18069-18077.

25. Travis MA, Sheppard D. TGF- $\beta$ activation and function in immunity. Annu Rev Immunol. 2014;32:51-82.

26. Spender LC, et al. TGF-beta induces apoptosis in human B cells by transcriptional regulation of BIK and BCL-XL. Cell Death Differ. 2009;16(4):593-602.

27. Lebman DA, Edmiston JS. The role of TGF-beta in growth, differentiation, and maturation of B lymphocytes. Microbes Infect. 1999;1(15):1297-1304

28. Gros MJ, Naquet P, Guinamard RR. Cell intrinsic TGF-beta 1 regulation of B cells. J Immunol. 2008;180(12):8153-8158.

29. Cerutti A. The regulation of IgA class switching. Nat Rev Immunol. 2008;8(6):421-434.

30. Cazac BB, Roes J. TGF-beta receptor controls B cell responsiveness and induction of IgA in vivo. Immunity. 2000;13(4):443-451.

31. Goodnow CC, et al. Self-tolerance checkpoints in B lymphocyte development. Adv Immunol. 1995;59:279-368.

32. William J, Euler C, Primarolo N, Shlomchik MJ. B cell tolerance checkpoints that restrict pathways of antigen-driven differentiation. J Immunol. 2006;176(4):2142-2151.

33. Culton DA, et al. Early preplasma cells define a tolerance checkpoint for autoreactive B cells. J Immunol. 2006;176(2):790-802

34. Hahn SA, et al. Soluble GARP has potent antiinflammatory and immunomodulatory impact on human CD4 ${ }^{+}$T cells. Blood. 2013;122(7):1182-1191.

35. Meyer-Martin H, et al. GARP inhibits allergic airway inflammation in a humanized mouse model. Allergy. 2016;71(9):1274-1283.

36. Liénart S, Stockis J, Dedobbeleer O, Lucas S. Targeting immunosuppression by Tregs with monoclonal antibodies against GARP. Oncoimmunology. 2016;5(3):e1074379.

37. Bekeredjian-Ding I, Jego G. Toll-like receptors--sentries in the B-cell response. Immunology. 2009;128(3):311-323.

38. Pasare C, Medzhitov R. Control of B-cell responses by Toll-like receptors. Nature. 2005;438(7066):364-368.

39. Liu B, Dai J, Zheng H, Stoilova D, Sun S, Li Z. Cell surface expression of an endoplasmic reticulum resident heat shock protein gp96 triggers MyD88-dependent systemic autoimmune diseases. Proc Natl Acad Sci USA. 2003;100(26):15824-15829.

40. Chen JQ, Szodoray P, Zeher M. Toll-like receptor pathways in autoimmune diseases. Clin Rev Allergy Immunol. 2016;50(1):1-17. 
41. Farrugia M, Baron B. The role of Toll-like receptors in autoimmune diseases through failure of the self-recognition mechanism. Int J Inflam. 2017;2017:8391230.

42. Theofilopoulos AN. TLRs and IFNs: critical pieces of the autoimmunity puzzle. J Clin Invest. 2012;122(10):3464-3466.

43. Marshak-Rothstein A. Toll-like receptors in systemic autoimmune disease. Nat Rev Immunol. 2006;6(11):823-835.

44. Dedobbeleer O, Stockis J, van der Woning B, Coulie PG, Lucas S. Cutting edge: Active TGF- $\beta 1$ released from GARP/TGF$\beta 1$ complexes on the surface of stimulated human B lymphocytes increases class-switch recombination and production of IgA. J Immunol. 2017;199(2):391-396.

45. Wu BX, et al. Glycoprotein A repetitions predominant (GARP) positively regulates transforming growth factor (TGF) $\beta 3$ and is essential for mouse palatogenesis. J Biol Chem. 2017;292(44):18091-18097.

46. Roes J, Choi BK, Cazac BB. Redirection of B cell responsiveness by transforming growth factor beta receptor. Proc Natl Acad Sci USA. 2003;100(12):7241-7246.

47. Ollendorff V, Szepetowski P, Mattei MG, Gaudray P, Birnbaum D. New gene in the homologous human 11q13-q14 and mouse 7F chromosomal regions. Mamm Genome. 1992;2(3):195-200.

48. Wu HJ, Wu E. The role of gut microbiota in immune homeostasis and autoimmunity. Gut Microbes. 2012;3(1):4-14.

49. Mu Q, Kirby J, Reilly CM, Luo XM. Leaky gut as a danger signal for autoimmune diseases. Front Immunol. 2017;8:598.

50. Chistiakov DA, Bobryshev YV, Kozarov E, Sobenin IA, Orekhov AN. Intestinal mucosal tolerance and impact of gut microbiota to mucosal tolerance. Front Microbiol. 2014;5:781.

51. Lee JH, Wang LC, Lin YT, Yang YH, Lin DT, Chiang BL. Inverse correlation between CD4+ regulatory T-cell population and autoantibody levels in paediatric patients with systemic lupus erythematosus. Immunology. 2006;117(2):280-286.

52. Sage PT, Sharpe AH. T follicular regulatory cells in the regulation of B cell responses. Trends Immunol. 2015;36(7):410-418.

53. Perry D, Sang A, Yin Y, Zheng YY, Morel L. Murine models of systemic lupus erythematosus. J Biomed Biotechnol. 2011;2011:271694.

54. Su DL, Lu ZM, Shen MN, Li X, Sun LY. Roles of pro- and anti-inflammatory cytokines in the pathogenesis of SLE. J Biomed Biotechnol. 2012;2012:347141.

55. Saxena V, et al. Dual roles of immunoregulatory cytokine TGF-beta in the pathogenesis of autoimmunity-mediated organ damage. J Immunol. 2008;180(3):1903-1912.

56. Zhuang H, Szeto C, Han S, Yang L, Reeves WH. Animal models of interferon signature positive lupus. Front Immunol. 2015;6:291.

57. Xu Y, Zeumer L, Reeves WH, Morel L. Induced murine models of systemic lupus erythematosus. Methods Mol Biol. 2014;1134:103-130

58. Manolova I, Gerenova J, Ivanova M. Serum levels of transforming growth factor- $\beta 1$ (TGF- $\beta 1$ ) in patients with systemic lupus erythematosus and Hashimoto's thyroiditis. Eur Cytokine Netw. 2013;24(1):69-74.

59. Ohtsuka K, Gray JD, Stimmler MM, Toro B, Horwitz DA. Decreased production of TGF-beta by lymphocytes from patients with systemic lupus erythematosus. J Immunol. 1998;160(5):2539-2545.

60. Khalil AM, Cambier JC, Shlomchik MJ. B cell receptor signal transduction in the GC is short-circuited by high phosphatase activity. Science. 2012;336(6085):1178-1181.

61. Gonnella PA, Waldner HP, Weiner HL. B cell-deficient (mu MT) mice have alterations in the cytokine microenvironment of the gut-associated lymphoid tissue (GALT) and a defect in the low dose mechanism of oral tolerance. J Immunol. 2001;166(7):4456-4464.

62. Tsitoura DC, Yeung VP, DeKruyff RH, Umetsu DT. Critical role of B cells in the development of T cell tolerance to aeroallergens. Int Immunol. 2002;14(6):659-667.

63. Song F, et al. The Peyer's patch is a critical immunoregulatory site for mucosal tolerance in experimental autoimmune encephalomylelitis (EAE). J Autoimmun. 2008;30(4):230-237.

64. Tokuyama H, Tokuyama Y. The regulatory effects of all-trans-retinoic acid on isotype switching: retinoic acid induces IgA switch rearrangement in cooperation with IL-5 and inhibits IgG1 switching. Cell Immunol. 1999;192(1):41-47.

65. Snapper CM, Waegell W, Beernink H, Dasch JR. Transforming growth factor-beta 1 is required for secretion of IgG of all subclasses by LPS-activated murine B cells in vitro. J Immunol. 1993;151(9):4625-4636.

66. Zan H, Cerutti A, Dramitinos P, Schaffer A, Casali P. CD40 engagement triggers switching to IgA1 and IgA2 in human B cells through induction of endogenous TGF-beta: evidence for TGF-beta but not IL-10-dependent direct $\mathrm{S}$ mu-->S alpha and sequential S mu-->S gamma, S gamma-->S alpha DNA recombination. J Immunol. 1998;161(10):5217-5225.

67. Fujihashi K, et al. Peyer's patches are required for oral tolerance to proteins. Proc Natl Acad Sci USA. 2001;98(6):3310-3315.

68. Kweon MN, et al. Mucosally induced systemic T cell unresponsiveness to ovalbumin requires CD40 ligand-CD40 interactions. J Immunol. 1999;162(4):1904-1909.

69. Sun JB, Flach CF, Czerkinsky C, Holmgren J. B lymphocytes promote expansion of regulatory T cells in oral tolerance: powerful induction by antigen coupled to cholera toxin B subunit. J Immunol. 2008;181(12):8278-8287.

70. Pandey S, Kawai T, Akira S. Microbial sensing by Toll-like receptors and intracellular nucleic acid sensors. Cold Spring Harb Perspect Biol. 2014;7(1):a016246.

71. Weltzin R, Lucia-Jandris P, Michetti P, Fields BN, Kraehenbuhl JP, Neutra MR. Binding and transepithelial transport of immunoglobulins by intestinal M cells: demonstration using monoclonal IgA antibodies against enteric viral proteins. J Cell Biol. 1989;108(5):1673-1685.

72. Apter FM, Michetti P, Winner LS, Mack JA, Mekalanos JJ, Neutra MR. Analysis of the roles of antilipopolysaccharide and anti-cholera toxin immunoglobulin A (IgA) antibodies in protection against Vibrio cholerae and cholera toxin by use of monoclonal IgA antibodies in vivo. Infect Immun. 1993;61(12):5279-5285.

73. Mantis NJ, Rol N, Corthésy B. Secretory IgA's complex roles in immunity and mucosal homeostasis in the gut. Mucosal Immunol. 2011;4(6):603-611.

74. Shi N, Li N, Duan X, Niu H. Interaction between the gut microbiome and mucosal immune system. Mil Med Res. 2017;4:14.

75. Meng W, et al. An atlas of B-cell clonal distribution in the human body. Nat Biotechnol. 2017;35(9):879-884

76. Bonder MJ, et al. The effect of host genetics on the gut microbiome. Nat Genet. 2016;48(11):1407-1412. 
77. Jostins L, et al. Host-microbe interactions have shaped the genetic architecture of inflammatory bowel disease. Nature. 2012;491(7422):119-124.

78. Bønnelykke K, et al. Meta-analysis of genome-wide association studies identifies ten loci influencing allergic sensitization. Nat Genet. 2013;45(8):902-906.

79. Ma K, Li J, Fang Y, Lu L. Roles of B cell-intrinsic TLR signals in systemic lupus erythematosus. Int J Mol Sci. 2015;16(6):13084-13105.

80. Nickerson KM, et al. TLR9 regulates TLR7- and MyD88-dependent autoantibody production and disease in a murine model of lupus. J Immunol. 2010;184(4):1840-1848.

81. Fujio K, Okamura T, Sumitomo S, Yamamoto K. Therapeutic potential of regulatory cytokines that target B cells. Int Immunol. 2016;28(4):189-195.

82. Haupt S, Söntgerath VS, Leipe J, Schulze-Koops H, Skapenko A. Methylation of an intragenic alternative promoter regulates transcription of GARP. Biochim Biophys Acta. 2016;1859(2):223-234.

83. Shah S, Qiao L. Resting B cells expand a CD4+CD25+Foxp3+ Treg population via TGF-beta3. Eur J Immunol. 2008;38(9):2488-2498

84. Kaminski DA, Letterio JJ, Burrows PD. Differential regulation of mouse B cell development by transforming growth factor beta1. Dev Immunol. 2002;9(2):85-95.

85. Parekh VV, Prasad DV, Banerjee PP, Joshi BN, Kumar A, Mishra GC. B cells activated by lipopolysaccharide, but not by anti-Ig and anti-CD40 antibody, induce anergy in CD8+ T cells: role of TGF-beta 1. J Immunol. 2003;170(12):5897-5911.

86. Tanaka KF, et al. Flexible accelerated STOP tetracycline operator-knockin (FAST): a versatile and efficient new gene modulating system. Biol Psychiatry. 2010;67(8):770-773.

87. Dai J, Liu B, Ngoi SM, Sun S, Vella AT, Li Z. TLR4 hyperresponsiveness via cell surface expression of heat shock protein gp96 potentiates suppressive function of regulatory T cells. J Immunol. 2007;178(5):3219-3225.

88. Liu B, et al. TLR4 up-regulation at protein or gene level is pathogenic for lupus-like autoimmune disease. J Immunol. 2006;177(10):6880-6888. 